\title{
Expression of the pair-rule gene homologs runt, Pax3/7, even-skipped-1 and even-skipped-2 during larval and juvenile development of the polychaete annelid Capitella teleta does not support a role in segmentation
}

\author{
Elaine C Seaver ${ }^{1 *}$, Emi Yamaguchi ${ }^{1}$, Gemma S Richards $^{2}$ and Néva P Meyer ${ }^{3}$
}

\begin{abstract}
Background: Annelids and arthropods each possess a segmented body. Whether this similarity represents an evolutionary convergence or inheritance from a common segmented ancestor is the subject of ongoing investigation.

Methods: To investigate whether annelids and arthropods share molecular components that control segmentation, we isolated orthologs of the Drosophila melanogaster pair-rule genes, runt, paired (Pax3/7) and eve, from the polychaete annelid Capitella teleta and used whole mount in situ hybridization to characterize their expression patterns.

Results: When segments first appear, expression of the single C. teleta runt ortholog is only detected in the brain. Later, Ct-runt is expressed in the ventral nerve cord, foregut and hindgut. Analysis of Pax genes in the C. teleta genome reveals the presence of a single Pax3/7 ortholog. Ct-Pax3/7 is initially detected in the mid-body prior to segmentation, but is restricted to two longitudinal bands in the ventral ectoderm. Each of the two C. teleta eve orthologs has a unique and complex expression pattern, although there is partial overlap in several tissues. Prior to and during segment formation, Ct-eve1 and Ct-eve2 are both expressed in the bilaterial pair of mesoteloblasts, while Ct-eve1 is expressed in the descendant mesodermal band cells. At later stages, Ct-eve2 is expressed in the central and peripheral nervous system, and in mesoderm along the dorsal midline. In late stage larvae and adults, Ct-eve1 and Ct-eve2 are expressed in the posterior growth zone.

Conclusions: C. teleta eve, Pax3/7 and runt homologs all have distinct expression patterns and share expression domains with homologs from other bilaterians. None of the pair-rule orthologs examined in C. teleta exhibit segmental or pair-rule stripes of expression in the ectoderm or mesoderm, consistent with an independent origin of segmentation between annelids and arthropods.
\end{abstract}

Keywords: Capitella, Eve, Pax3/7, polychaete, Runt, Segmentation

\section{Background}

The evolution of segmentation in bilaterian animals is an ongoing area of investigation and debate (reviewed in [1-4]). Three major animal clades contain segmented representatives, the Annelida, the Arthropoda and the Chordata. According to molecular phylogenies [5,6], each

\footnotetext{
* Correspondence: seaver@hawaii.edu

${ }^{1}$ Kewalo Marine Laboratory, Pacific Biosciences Research Center, University of Hawaii, 41 Ahui Street, Honolulu, HI, USA

Full list of author information is available at the end of the article
}

of these clades is more closely related to animals that lack a segmented body than to each other. Thus, the question remains whether segmentation arose independently in distinct lineages, or whether most extant clades lost the segmented body plan that was present in a common segmented ancestor. One approach to address this question is to compare the molecular mechanisms controlling segment generation across taxa, with the assumption that shared molecular mechanisms reflect a common evolutionary history. 
Segment formation is best understood in Drosophila melanogaster, in which a detailed understanding of the genetic control of segmentation has served as a framework for comparative studies in other arthropods. Briefly, a segmentation gene cascade sequentially divides the embryo into smaller units through the hierarchical action of the gap, pair-rule and segment polarity genes (reviewed in [7]). The pair-rule genes are the first to be expressed in a periodic pattern in D. melanogaster, and include the genes paired (Pax3/7), even-skipped (eve) and runt. These genes are expressed with a two-segment periodicity in ectodermal stripes along the anterior-posterior axis. Mutants of pair-rule genes lack alternating segmental structures such as denticle bands in the larval cuticle [8]. In contrast, mutants in segment polarity genes exhibit defects in the pattern of every segment.

The mode of segmentation in D. melanogaster is derived compared to other arthropods (reviewed in [9]). In $D$. melanogaster, all segments form at virtually the same time (long germ band development) and much of the patterning critical for segment formation occurs prior to cellularization. In most other arthropods, segments form in a temporal progression from anterior to posterior and the earliest segments form before the tissue for additional segments is present (short germ band development). Although many insects also develop via an early syncytial stage, this is a derived feature within arthropods, and many arthropods form all or most segments within a cellular environment.

The expression patterns of pair-rule and segment polarity gene orthologs have been examined across arthropods, including in insects, and also in chelicerates, myriapods and crustaceans. In general, pair-rule genes show more variability in their expression patterns than do the highly conserved segment polarity genes, and there are examples lacking periodic expression patterns even within insects [10]. In several arthropods, pair-rule gene orthologs are expressed in stripes in every segment; in some cases the onset of expression is clearly prior to segment generation. This pattern is more consistent with a segment polarity role than a pair-rule function. For example, in the centipede Lithobius atkinsoni [11] and the spider Cupiennius salei [12], Pax3/7 is expressed in a portion of every segment. In the spider mite Tetranychus urticae [13] and the millipede Glomeris marginata [14], runt has a segmental pattern. Likewise, eve is expressed with a segmental periodicity in L. atkinsoni [15], C. salei [16] and in the insect Oncopeltus fasciatus [17]. In D. melanogaster it is notable that several pair-rule genes, including even-skipped, runt and paired, have an initial pair-rule expression pattern of seven alternating stripes that later matures into a segmental 14-stripe pattern [18-20]. These data suggest that the pair-rule orthologs have a general, and likely ancestral, function in arthropod segment formation. The extent to which pair-rule patterning is conserved across arthropods, and whether pair-rule patterning was utilized primarily in holometabolous insects (flies, bees, beetles and moths) or ancestrally at the base of arthropods, remains an open question $[7,11,18]$. Regardless, the pair-rule genes are useful markers for inter-taxonomic comparisons to reconstruct the evolution of segmentation.

The mechanistic understanding of the molecular control of segment formation in annelids is poor compared to that in chordates and arthropods. Efforts to identify genes involved in annelid segmentation have largely utilized a candidate gene approach. Examining the expression of genes involved in arthropod segmentation and vertebrate somitogenesis and identification of shared components of a common genetic program for segment formation would support a shared evolutionary origin of segmentation. Expression patterns of segment polarity gene orthologs have been examined in a number of annelid species; the most well-characterized is the segment polarity gene engrailed. In contrast to the highly conserved expression pattern of en across arthropods [15,21-23], en expression patterns among annelids exhibit substantial variability. Although en is expressed in ectodermal stripes in the polychaete annelid Platynereis dumerilii [24], this pattern is not apparent in any other annelid examined, including in the polychaetes Chaetopterus sp. [25], Hydroides elegans and Capitella teleta [26] Blake, Grassle \& Eckelbarger, 2009 [27] (previously known as Capitella sp. I) and in the leech Helobdella triserialis [28]. Thus, there is currently a discrepancy in en expression patterns among annelids and the $P$. dumerilii pattern may represent a convergence, rather than a common origin, with the arthropod pattern. Alternatively, if the P. dumerilii and arthropod patterns represent a conservation, there has been extensive divergence in en expression among annelids.

Previously, we investigated the evolution of bilaterian segmentation by characterizing the expression of orthologs of the segment polarity genes, en, $w g$ and $h h$ [26] and of the pair-rule genes hairy [29] and odd-paired [30] in C. teleta. Here, we report the expression of the pair-rule gene orthologs runt, paired (also called pax group III or Pax3/ 7) and two eve genes in C. teleta immediately prior to and during larval segment formation. Additionally, we characterize $\mathrm{Pax} 3 / 7$ and eve expression during adult segment formation. C. teleta eve, Pax3/7 and runt genes lack segmental or pair-rule stripes of expression in both the ectoderm and mesoderm, even though Ct-Pax3/7, Ct-eve1 and Ct-eve 2 expression is initiated prior to overt segmentation. Each ortholog examined has a distinct expression pattern and exhibits expression domains conserved with those found in other bilaterians. 


\section{Methods}

\section{Animal husbandry}

A C. teleta colony was maintained in the laboratory according to published culturing methods [31]. Embryos and larvae were recovered as previously described [32].

\section{Cloning and sequencing of $C$. teleta runt, $\mathrm{Pax} 3 / 7$, and eve orthologs}

Fragments corresponding to conserved regions of runt, Pax3/7 and eve orthologs were isolated from C. teleta by degenerate polymerase chain reaction (PCR) using a cDNA template prepared from mixed embryonic and larval stages. To recover a fragment of runt, two rounds of amplification were performed in a semi-nested PCR reaction using the following primers from published sequences [33]: runfw-1: 5' - RCNRYNATGAARAAYCARGTNGC -3' and runbw: 5'-CKNGGYTCNCKNGGNCCRTC-3' followed by runfw-2: 5'-MRNTTYAAYGAYYTNMGNTTYGTNGG -3' and runbw. To isolate a fragment of Pax3/ 7 from mixed stage cDNA, a semi-nested approach was utilized with the following published primers [34] representing the conserved paired domain and paired-like homeodomain: Prbyfout: 5'- GGNGGNGTNTTYATH AAYGG -3' and Prbyr: 5'-RTTNSWRAACCANACYTG -3' followed by Prbyfin: 5'-MARATHGTNGARATGGC -3 ' and Prbyr in a second round of amplification. A 226 base pair (bp) fragment of eve was recovered from a genomic DNA template using the following degenerate primers: ab-evefw: 5'-MGTTAYMGTACIGCITTYAC-3' and eve-bw1: 5'-CKYTGNCKYTTRTCYTTCAT-3' [33]. Additional sequences for Ct-eve, Ct-Pax3/7 and Ct-runt were obtained from a mixed stage embryonic and larval stage template using gene-specific primers with the Smart rapid amplification of cDNA ends (RACE) amplification kit (Clontech, Mountain View, CA, USA). Resulting fragments were cloned into the pGEM-T Easy vector (Promega, Fitchburg, WI, USA) and sequenced by the University of Hawaii sequencing facility (Honolulu, HI, USA) or by Macrogen Inc. (Seoul, South Korea). The resulting products were: a 702 bp 5' RACE fragment and a 1269 bp 3' RACE fragment for Ct-Pax3/7, a 644 bp 5' RACE fragment and a 945 bp 3' RACE fragment for Ct-runt. For eve, a 460 bp 5' RACE fragment was recovered by PCR using genespecific primers. Sequencing of multiple cDNA clones from 5' RACE reactions revealed the presence of a five amino acid insertion in some clones. Two distinct 3' RACE fragments were recovered: a 583 bp and a 734 bp fragment. Gene-specific primers designed to each of the 5' and 3' eve sequence variants were used to amplify and characterize features of individual cDNAs. Subsequent to the isolation of Ct-runt, Ct-Pax3/7,Ct-eve1 and Ct-eve 2 by degenerate PCR, sequences became available for the C. teleta genome project (Department of Energy, Joint Genome Institute, Walnut Creek, CA, USA [35]). Cloned sequences were consistent with predicted gene models from the genome. The genome was searched for additional runt, $\mathrm{Pax3} / 7$ and eve paralogs; none were detected. Linkage analysis of $\mathrm{Ct}$ eve1 and Ct-eve2 genes was performed through searches of the C. teleta genome using nucleotide sequences obtained from degenerate and RACE PCR clones. Accession numbers for Ct-runt, Ct-eve1, Ct-eve2 and Ct-Pax3/7 are listed in Additional file 1: Table S1.

\section{Sequence alignments and phylogenetic analyses}

Sequences isolated by degenerate PCR and RACE were assigned putative orthologies based on BLASTX searches of the GenBank database from the National Center for Biotechnology Information. In addition, tBLASTn searches of the C. teleta genome (Joint Genome Institute (JGI), Walnut Creek, CA, USA) were conducted to find all homologs of eve, runt and the paired homeobox (pax) family. Two putative orthologs were found for eve, one for runt and six for pax family members. Amino acid sequences from diverse animal taxa were downloaded from the protein database in GenBank. Alignments of conserved domains were generated with ClustalX, using default parameters in MacVector 11.1.1 (MacVector, Inc., Cary, NC, USA). The domains analyzed were the homeodomain and 10 amino acids flanking the 5'- and 3'-ends of the homeodomain for Eve, the runt domain for Runt and the 127 amino acid paired domain for the Pax family. Alignments were edited by hand to correct obvious alignment errors. Nexus alignments are available upon request. Sequences, abbreviations and accession numbers used are included in Additional file 1: Table S1.

ProtTest v2.4 [36] was run for each alignment to determine the appropriate model of protein evolution. The Jones model was recommended and used for the Eve and the Pax family, and the RtRev model for Runt. Both Bayesian and maximum likelihood methods were used for each gene family. Bayesian analysis was conducted using MrBayes v3.1.2 [37], with the model determined by ProtTest. For the Runt and Pax alignments, three million generations were run, sampled every 100 generations, with four independent runs and four chains. Ten million generations were run for the Eve alignment. Once convergence was reached, majority rule consensus trees were generated with burnin values of 25,000 (Eve), 9,700 (Runt) and 5,400 (Pax). Maximum likelihood analyses were performed with RAxML v7.0.0 [38] using the same models as for Bayesian analysis with 1,000 bootstrap replicates. Trees were visualized with FigTree v1.3.1 [39] and drawn using Adobe Illustrator version CS4.

\section{Whole mount in situ hybridization}

Whole mount in situ hybridization was performed according to previously published protocols [26]. Digoxigeninlabeled riboprobes (dig-11-UTP; Roche Diagnostics, 
Indianapolis, IN, USA) were transcribed with the MEGAscript High Yield Transcription Kit (Ambion, Austin, TX, USA) and diluted to working concentrations of $3 \mathrm{ng} / \mu \mathrm{L}$ for Ct-runt, $0.25 \mathrm{ng} / \mu \mathrm{L}$ for $C t-P a x 3 / 7,0.1 \mathrm{ng} / \mu \mathrm{L}$ for $C t$ eve1 and $0.2 \mathrm{ng} / \mu \mathrm{L}$ for $C t$-eve2. Probe lengths were as follows: 1041 bp for Ct-runt, 1270 bp for Ct-Pax3/7, 1379 bp for Ct-eve1 and $1224 \mathrm{bp}$ for Ct-eve2. Following termination of the development reaction, specimens were equilibrated and stored in $80 \%$ glycerol, $20 \% 5 \times$ phosphate buffered saline (PBS). The detailed protocol is available upon request.

\section{Visualization of mid-body mesoderm}

Animals were fixed and labeled according to previously described conditions (fixation \#4, [40]). Specimens were exposed to a 1:500 dilution of mouse anti-histone antibody (F152, C25.WJJ; Millipore, Billerica, MA, USA) and a 1:400 dilution of goat anti-mouse rhodamine (Invitrogen, Carlsbad, CA, USA). After antibody labeling, animals were incubated in 1:100 BODIPY FL phallacidin (Invitrogen) in PBS, $0.1 \%$ Triton X-100 (PBT) for two hours at room temperature followed by several PBT washes over 45 minutes. Animals were mounted in Slow Fade Gold (Invitrogen) and analyzed.

\section{Microscopy}

Riboprobe-labeled specimens were analyzed using differential interference contrast (DIC) optics on an Axioskop 2 compound microscope (Zeiss, Munich, Germany). Digital images were captured with either a stem-mounted 4.0 megapixel Nikon Coolpix 4500 (Nikon, Inc., Melville, NY, USA) or SpotFlex digital camera (Diagnostic Instruments, Inc., Sterling Heights, MI, USA). Confocal laser scanning microscopy was performed using an Axioplan 2 LSM510 microscope (Zeiss). Three-dimensional reconstructions were generated from confocal images with ImageJ (National Institutes of Health, Bethesda, MD, USA). Figures and diagrams were constructed using Photoshop CS4 and Illustrator CS4 (Adobe Systems Inc., New York, NY, USA). Some panels in figures seven and eight (see Figure legends) are composites of multiple DIC focal planes that were merged using Helicon Focus (Helicon Soft Ltd., Kharkov, Ukraine).

\section{Results \\ Cloning and phylogenetic analyses of $C$. teleta runt, eve and $P a \times 3 / 7$ genes}

Using degenerate and RACE PCR, we isolated fragments comprising 1585 bp of a C. teleta runt ortholog, designated Ct-runt. The sequence contained a 157 bp 5' untranslated region (UTR), a $570 \mathrm{bp}$ open-reading frame (ORF) predicting a protein of 190 amino acids and an 857 bp 3' UTR and poly-A tail. The predicted ORF included a conserved Runt DNA binding domain of 128 amino acids.
When compared to Runt sequences from other organisms, the Ct-runt predicted ORF showed strong conservation of amino acid residues throughout the Runt DNA binding domain (Figure 1A). Phylogenetic analyses of the Runt domain from Ct-Runt were performed using both Bayesian and maximum likelihood methods. Ct-Runt groups most closely with the Lozenge protein from the parasitic flatworm Schistosoma mansoni, another lophotrochozoan, and these two sequences fall within a group that contains ecdysozoan, lophotrochozoan and non-vertebrate deuterostome Runt sequences (Additional file 2: Figure S1). The vertebrate and cephalochordate Runt and Runx sequences form a well-supported separate group, and cnidarian sequences fall outside of both groups.

The C. teleta runt ortholog we recovered lacks the characteristic motif VWRPY at the C-terminal end, which is necessary for Groucho-mediated transcriptional repression activity [41]. To confirm that the missing motif was not due to a cloning artifact, 3' RACE PCR was repeated with a second primer set and an independently generated cDNA pool. The resulting fragment was identical in sequence to the original Ct-runt 3' RACE product. In addition, we identified a single runt sequence from searches of the C. teleta genome [35], which has the same amino acid sequence as the sequence isolated by PCR and lacks the C-terminal Groucho-binding domain. Searches of the Lottia gigantea genome [42] reveal that its predicted runt homolog also lacks the VWRPY C-terminal motif (Protein ID 119594).

A paired $(\mathrm{Pax} 3 / 7)$ ortholog, $\mathrm{Ct}-\mathrm{Pax} 3 / 7$, was amplified by degenerate and RACE PCR from a mixed stage cDNA template. This sequence was composed of a 99 bp 5' UTR, a 1536 bp ORF predicting a protein of 416 amino acids and a 175 bp 3' UTR that includes a poly-A tail. Pax3/7 proteins are defined by the presence of three conserved domains: a paired domain and 'paired' class homeodomain, both of which are DNA binding domains, and a short octapeptide motif, which is typically located in the linker region between the paired domain and the homeodomain. This octapeptide motif has been shown to act as a site for Groucho-mediated repression [43]. The predicted Ct-Pax3/7 ORF from the C. teleta cDNA contains a putative paired domain and a 'paired' class homeodomain (Figure 1B) but lacks the octapeptide motif in the linker region. To confirm our placement of $\mathrm{Ct}-\mathrm{Pax} 3 / 7$ within the Pax3/7 family, we identified other putative $\mathrm{Pax}$ homologs in the $C$. teleta genome and ran phylogenetic analyses using the paired domain. Several distinct Pax families are broadly distributed across bilaterians and include: Pax1/9, Pax2/5/8, Pax3/7, Pax4/6 and Pox neuro [44]. More recently, a Pax $\beta$ family has been described, a novel Pax subfamily only found in lophotrochozoans [45]. C. teleta has six Pax family members, with a single representative in each of the Pax families Pox neuro, Pax $\beta$, Pax1/9, Pax2/5/ 


\begin{tabular}{|c|c|}
\hline runt domain & \\
\hline Ct-Runt & TLSAVLSEHPGELVRTGSPNFVCSVLPSHWRSNKTLPVSFKVVALGEVKDGTKVTLNVGNDENC \\
\hline BlaRunt & G-VDA-AD - - - - - D - - \\
\hline DmeRunt & S-HEM-Q-YH- - AQ - - - -SIL- -A - -N- - - - -S- - GA - - - I- - DD - P- - - L-SIKC- - - - Y \\
\hline DmeLozenge & 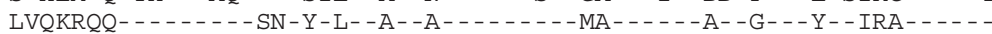 \\
\hline MmuRunx1 & SMVE- - AD - - - - - D- - - L \\
\hline MmuRunx2 & -MVEIIAD-A- \\
\hline MmuRunx3 & SMVD- -AD -A - - - - D - - - L- - - - - \\
\hline Ct-Runt & CGELRNAVTHMKNHVAKFNDLRFVGRSGRGKSFNLTI CVQTNPPQVATFOKA I KVTVDGPREPR \\
\hline BlaRunt & SA- - -NQAV - - Q - - R- - \\
\hline DmeRunt & - \\
\hline Dmelozenge & -ADV- $\mathrm{F}$ T-Q $\mathrm{Q}-\mathrm{-}$ - -1 \\
\hline MmuRunx1 & SA- \\
\hline MmuRunx2 & SA- \\
\hline MmuRunx3 & SA- - - -SAV- - Q - - R- - \\
\hline
\end{tabular}

B. Pax3/7 paired domain

\begin{tabular}{|c|c|}
\hline$t-1$ & GQGRVNQLGGVFINGRPLPNHIRLKI IEMASQGVRPCVISRTLRVSHGCVSKILQRYQETGSIR \\
\hline$\times 3 / 7$ & $---1-1$ \\
\hline & $-1-1$ \\
\hline & - \\
\hline$\times 3 / 7$ & 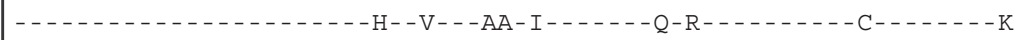 \\
\hline & - - - - - - - \\
\hline MmuPax7 & $-\mathrm{C}_{-1}$ \\
\hline Ct-P & PGSIGGSKPR . VATPDVEDRIHDLKKENPGIFSWEIRDRLLKDGVCDRSSVPSVSSISRVLRSH \\
\hline $3 / 7$ & $--\mathrm{A}-\cdots--,-\mathrm{KME}-\cdots-\mathrm{K}-\mathrm{EEY}---\mathrm{D}-\cdots--\mathrm{E}---\mathrm{E}-\mathrm{I}-\mathrm{EN}-\cdots \mathrm{LQKQT}$ \\
\hline DmeGs: & 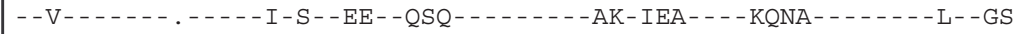 \\
\hline DmeGsbp & 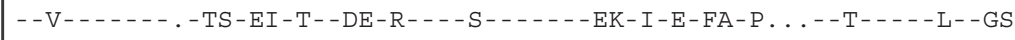 \\
\hline $3 / 7$ & 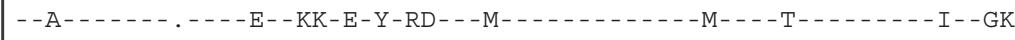 \\
\hline & --A- - - - - KQ - T- - - - KK-EEY-R-- - M- - - - - K- - GA - - -NT- - - - - - I - - K \\
\hline MmuPax7 & -A-1-1- \\
\hline
\end{tabular}

Pax3/7 homeodomain

\begin{tabular}{|c|c|}
\hline Ct $-\operatorname{Pax} 3 / 7$ & RKQRRSRTTFSADQLEHLEKAFDRTHYPDIYTREELAQRSGLTEARVQVWFSNRRARWRKQ \\
\hline $\mathrm{Hel} \mathrm{Pax} 3 / 7$ & 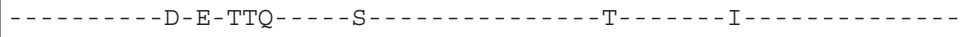 \\
\hline DmeGsbd & 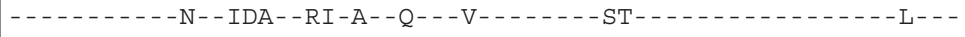 \\
\hline DmeGsbp & $---------\mathrm{T}-\mathrm{E}---\mathrm{A}--\mathrm{R}--\mathrm{S}--\mathrm{Q}---\mathrm{V}-------\mathrm{TTA}-----\mathrm{I}---------\mathrm{L}--\mathrm{H}$ \\
\hline $\mathrm{Bfl} \operatorname{Pax} 3 / 7$ & $---------\mathrm{TPE}---\mathrm{E}-----\mathrm{E}--\mathrm{H}------------\mathrm{TK}-----------------$ \\
\hline MmuPax3 & 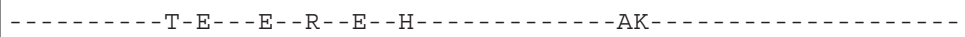 \\
\hline MmuPax7 & 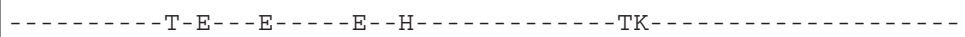 \\
\hline
\end{tabular}

\section{C. eve homeodomain}

\begin{tabular}{|c|c|}
\hline Ct-eve & IRRYRTAFTREQLGRLEREFLKENYVSRPRRCELAASLNLPESTIKVWFQNRRMKDKRQR \\
\hline Pdu-eve & 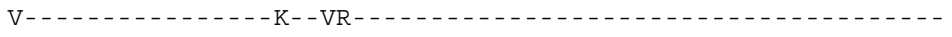 \\
\hline Hro-eve & L- - - - - - - - - AK $--\mathrm{K}----------------\mathrm{S} \mathrm{Q}-\mathrm{D}---\mathrm{C}----------------$ \\
\hline Iob-eve & L-- - - - - SKD - IS - - - K- - A- - - - I - - - K- - - - - TMG - - - - - - - - - - - - - - - \\
\hline Sam-eve & $-------------\mathrm{A}---\mathrm{K}--\mathrm{Y}--------------\mathrm{S} \mathrm{Q}-----------------------$ \\
\hline Dme-eve & 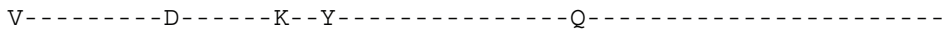 \\
\hline Bfl-evxl & 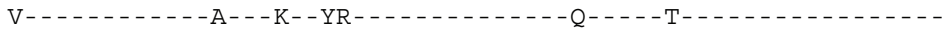 \\
\hline $\mathrm{Bfl}-\mathrm{evx} 2$ & V-------S---TA---K--HRD- - L- - - - - - - - A - - - - T- - - - - - - - - - - - \\
\hline Mmu-evx1 & M- - - - - - - - IA $---\mathrm{K}--$ YR $------------\mathrm{A}-----\mathrm{T}---------------$ \\
\hline Mmu-evx2 & 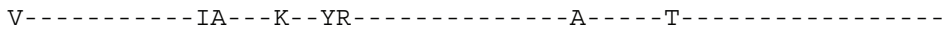 \\
\hline
\end{tabular}

Figure 1 Alignments of conserved domains in Ct-Runt, Ct-Pax3/7 and Ct-Eve. (A) Amino acid alignment of the Runt domain of Ct-Runt compared to the Runt domain of other animals. (B) Amino acid alignment of Ct-Pax3/7 and Pax3/7 proteins from other species. The alignments shown include the paired domain and the homeodomain. (C) Amino acid alignment of the Ct-Eve homeodomain with homeodomains from Eve proteins in a range of species. Dashes represent amino acid identities with the $C$. teleta sequences; dots represent gaps introduced by CLUSTALW to optimize alignments. Bfl: Branchiostoma floridae; Bla: B. lanceolatum; Dme: Drosophila melanogaster; Hel: Helobdella sp.; Hro: H. robusta; Mmu: Mus musculus; Pdu: Platynereis dumerilii; lob: llyanassa obsoleta; Sam: Schistocerca Americana. 
8, Pax3/7 and Pax4/6 (Figure 2). Both Bayesian posterior probability and maximum likelihood bootstrap show strong support for the distinct Pax gene groups. Ct-Pax3/7 groups most closely with the two Pax $3 / 7$ sequences from another annelid, the leech Helobdella sp. Austin. These lophotrochozoan Pax3/7 sequences group separately from the deuterostome Pax3 and Pax7 clade and the arthropod homologs Gooseberry, Pairberry and Paired.

A 226 bp fragment of eve was initially identified by degenerate PCR from a C. teleta genomic DNA template. Subsequent RACE PCR recovered a 5' fragment and two distinct 3' fragments. From sequence searches of the $C$. teleta genome, two distinct eve genes were identified, which we designate $C t$-eve 1 and $C t$-eve2. The Ct-eve1 and eve 2 predicted ORFs are 325 and 330 amino acids, respectively. The Ct-Eve1 and Ct-Eve2 predicted ORFs are largely identical within the predicted ORF; the exceptions are the two amino acids immediately 5' of the stop codon (KS for Ct-eve1 and PK for Ct-eve2), and a small five amino acid insertion present only in Ct-Eve2. This insertion immediately follows amino acid residue 85 and is located 17 amino acids 5' of the homeodomain. The homeodomain in both Eve sequences contains conserved amino acids characteristic of eve gene sequences (Figure 1C). Bayesian and maximum likelihood analyses of the homeodomain confirm the identity of Ct-eve1 and Cteve 2 as eve gene orthologs within the homeodomain superfamily (Additional file 3: Figure S2). Ct-Eve1 and Ct-Eve2 cluster together with Eve proteins from other animal taxa and separately from the homeodomain-containing Gbx and Engrailed outgroups. There is 100\% Bayesian posterior probability and $90 \%$ bootstrap support for the Eve node. Within the predicted ORFs, there are only seven nucleotide differences between $C t$-eve 1 and Ct-eve2, aside from sequence differences due to the five amino acid insertion in Ct-Eve 2 and the two amino acids immediately $5^{\prime}$ of the stop codon. In contrast, the two sequences diverge in sequence and length in the 3' UTR (Ct-eve1, $167 \mathrm{bp}$ and Ct-eve2, $349 \mathrm{bp}$ ).

Both Ct-eve1 and Ct-eve2 are located on scaffold 262, approximately $18.7 \mathrm{kbp}$ apart, and these two genes exhibit opposite transcriptional orientations (Figure 3). For both genes, the predicted ORFs are distributed among three exons, and the positions of the intron-exon boundaries are shared between the two genes. In contrast, the size and sequence composition of the first intron are different between Ct-eve1 (371 bp) and Ct-eve2 (652 bp). The second intron is the same in length and sequence in the two genes and is located within the homeodomain, between homeodomain positions 46 and 47. The position of the second intron within the homeodomain is conserved in the two amphioxus and mouse eve gene homologs and the single Tribolium castaneum eve gene [46,47], and is different from the position of the single intron in the
D. melanogaster eve gene, which is upstream of the homeodomain [48]. Exon 3 for both genes also contains 191 amino acids of the ORFs, as well as the gene-specific 3' UTRs. Comparisons of the $3 \mathrm{kbp}$ of 5' and 3' sequence flanking the two ORFs lack regions of sequence similarity between $C t$-eve 1 and $C t$-eve2. The C. teleta eve genes are not clustered with the Hox genes in the genome [49], although it remains a possibility that they are all on the same chromosome. In $P$. dumerilii, eve is on the same chromosome as the posterior class Hox gene Post2, but is not part of the Hox cluster [50], and T. castaneum eve is also not clustered with the Hox genes [47]. In contrast, eve orthologs in vertebrates and amphioxus, and in the cnidarian Nematostella vectensis, are clustered with the Hox genes [51-53].

\section{C. teleta development}

C. teleta development has been described previously and a standard staging system is used to distinguish among embryonic and larval stages (Figure 4 top) [32]. Following unequal spiral cleavage (Stages 1 to 2 ) and gastrulation (Stage 3), two trochal bands, the prototroch and telotroch, penetrate through the egg membrane to initiate the larval phase (Stages 4 to 9). The prototroch and telotroch delineate, respectively, the anterior and posterior boundaries of the segmented mid-body. The mouth is positioned immediately posterior of the prototroch, on the ventral side of the larva. The first four to five segments initially appear during early larval stages (Stage 5), and segmental furrows are visible on the ventral face of the larva $[28,32]$. The first ten segments form progressively from anterior to posterior within a 24 hour time frame, and three additional larval segments form from a posterior growth zone (PGZ) by late larval stages (Stage 9). The final three larval segments form at a rate of approximately one per day. Following metamorphosis, 40 to 50 additional posterior segments are generated from the PGZ.

The segmented ectoderm and mesoderm have distinct embryonic origins in C. teleta [54]. All segmental ectoderm is generated from the blastomere $2 \mathrm{~d}$, which also generates nonsegmental posterior ectoderm. The segmented mid-body ectoderm segregates from nonsegmental ectoderm within the first few divisions of $2 \mathrm{~d}$, such that $2 \mathrm{~d}^{11}$ generates segmented ectoderm and PGZ, but not the telotroch or the posterior unsegmented pygidium [54]. Most of the mid-body mesoderm arises from the two cells $3 \mathrm{c}$ and $3 \mathrm{~d}$, whose descendants form the right and left mesodermal bands, respectively (Figure 4). Initially, a pair of large posterior mesodermal cells is visible beneath the ectoderm (Figure 4A,D). Subsequently, two mesodermal bands become visible as a row of subsurface cells in the lateral part of the body (Figure 4B,C,E,F). Over time, the bands increase in cell number and expand 

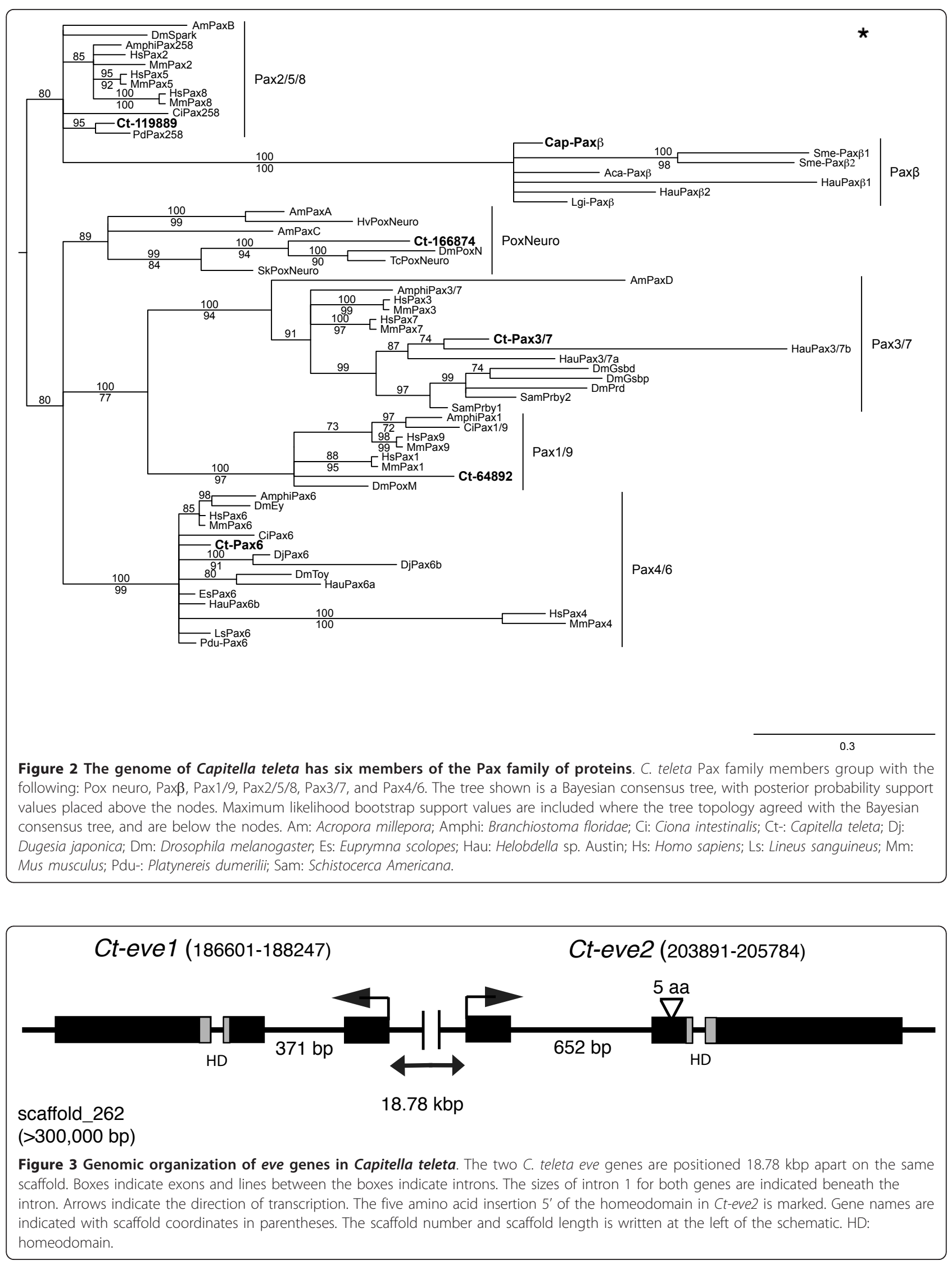


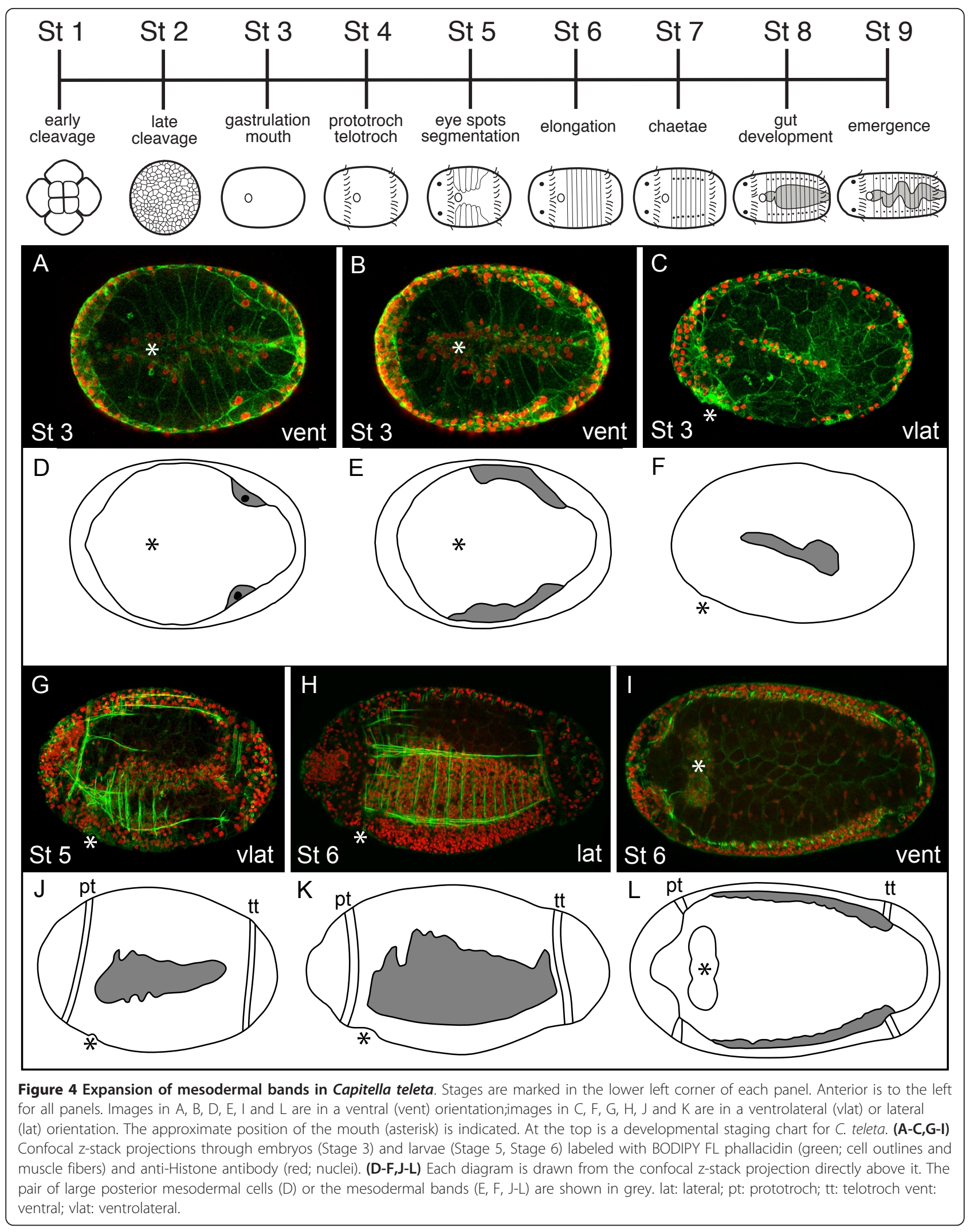


circumferentially (Figure 4G-L). By stage 5, when the first segments are visible, longitudinal and circular muscle fibers extend from the mesodermal bands (Figure 4G). Extension of a single circular muscle fiber per segment is one of the earliest signs of segmentation in the mesoderm and follows soon after ectodermal segmentation. These circular muscle fibers are positioned within the intersegmental grooves (Figure 4I) [32].

\section{Ct-runt expression}

Ct-runt is expressed in several distinct ectodermal tissues, predominantly in the nervous system, foregut and hindgut, but is never detected in ectodermal stripes of expression in the segmented part of the body (Figure 5). Most Ct-runt expression is transient, lasting only one to two days. Initially, Ct-runt expression is localized to two small domains in the brain in Stage 5 larvae (Figure 5A), and is not detected in Stage 3 and Stage 4 larvae. The first segments appear at Stage 5, and Ct-runt is not expressed in nascent segments or in the presumptive segmental tissue at this stage. At Stage 6, expression in the brain appears as several discrete cell clusters (Figure 5B,E). There is also expression in the anterior four to five segments in two columns of cells (Figure 5C). The more lateral runt-expressing cells are centrally positioned within the segment (Figure 5D). The medial cells are clearly in the forming ventral nerve cord. Because the lateral boundary of the ventral nerve cord is not yet distinct at this stage, it is unclear if the lateral cells (short arrows) are within or lateral to the ventral nerve cord. At Stage 7, several new domains of Ct-runt expression appear, most notably in the foregut. At late Stage 7, discrete sub-domains of the foregut become morphologically obvious, and there is expression of Ct-runt in the pharynx (Figure 5J). By Stage 8, foregut expression is no longer detectable. Expression also appears in the ventrolateral ectoderm of the PGZ during Stage 7 (Figure 5G, $\mathrm{I}, J$ ), which persists to Stage 8 (Figure $5 \mathrm{~K}$ ). At Stage 7, there is also transient expression in a small lateral mesodermal domain, posterior to the mouth (Figure $5 \mathrm{H}$ ). In the nervous system, expression in the ventral nerve cord expands to more posterior segments and is prominent in a single row of segmentally repeated cells (Figure 5F). Brain

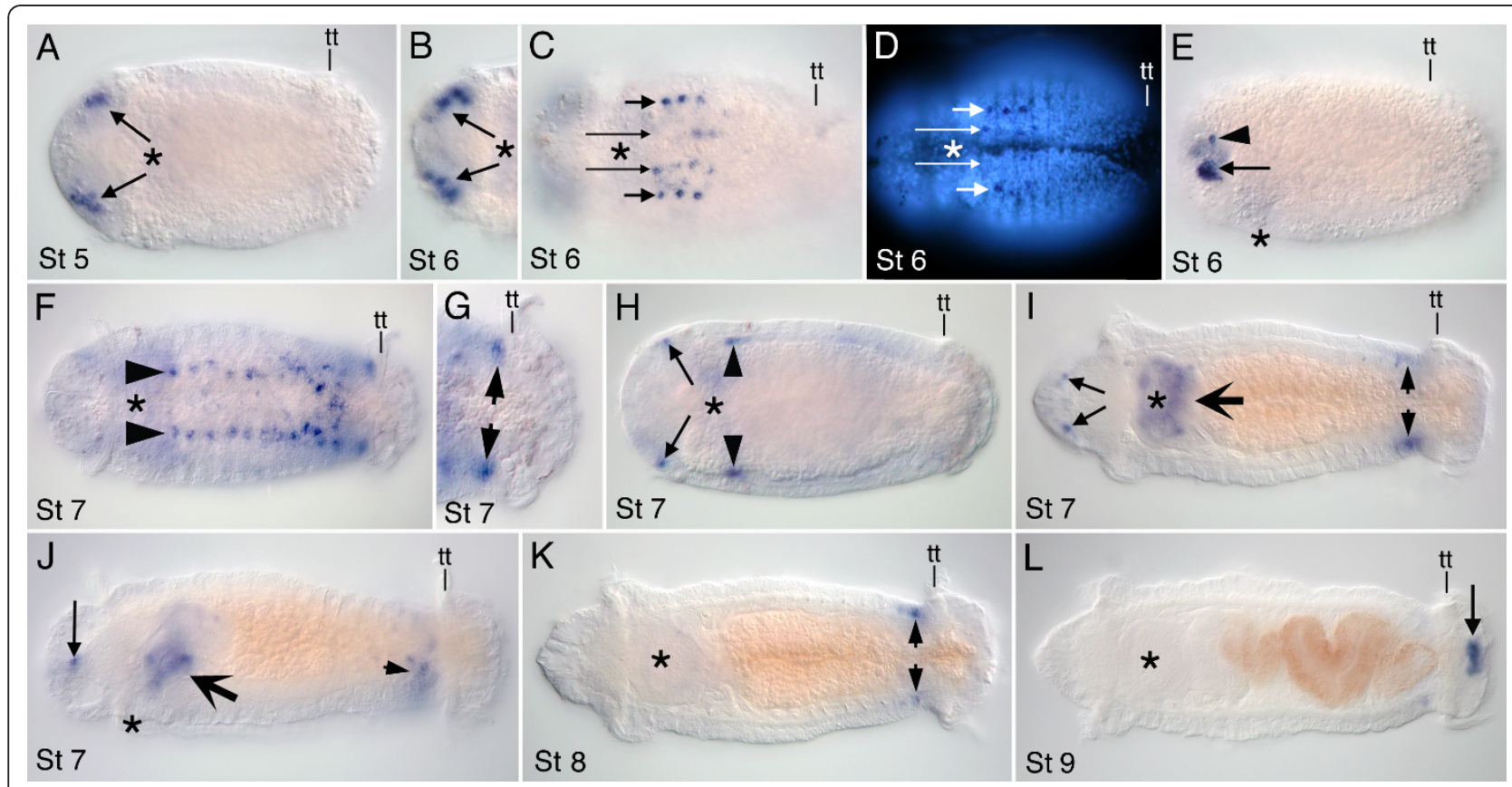

Figure $\mathbf{5}$ Larval expression of Ct-runt. Stages are marked in the lower left corner of each panel. Anterior is to the left for all panels. All panels are ventral views except for $\mathrm{E}$ and $\mathrm{J}$, which are lateral views with ventral down. An asterisk marks the position of the mouth. (A) $\mathrm{Ct}$-runt expression in the brain (arrows). (B,C) Two different focal planes of the same animal showing expression in the brain (B) and ventral nerve cord in anterior segments (long arrows in C). Short arrows point to expression in ventrolateral ectodermal cells. (D) Epi-fluorescence view showing position of medial (long arrows) and lateral-expressing cells (short arrows) within each segment. (E) Arrowhead and arrow mark discrete clusters of Ct-runt-expressing cells visible within a single focal plane in the brain. (F) Arrowheads show nervous system expression extending along the length of the ventral nerve cord. (G) Enlarged view of the posterior body of the larva shown in F. Arrows point to expression in the ectoderm of the PGZ. (H) Expression becomes restricted to a single small cluster in the brain (arrows) and in a small lateral domain of mesoderm (arrowheads). (I) At late Stage 7, Ct-runt is transiently expressed in the foregut (large arrow). There is also expression in the PGZ (short arrows) and the anterior head ectoderm (diagonal arrows). (J) Foregut expression is in the pharynx (large arrow). PGZ expression is ventrally localized (short arrow). Long arrow points to anterior head expression. (K) Expression is restricted to the PGZ at Stage 8 (short arrows). (L) Arrow indicates prominent expression in the hindgut of a late stage larva. tt: telotroch. 
expression ceases, although there remain Ct-runt-positive ectodermal cells in the head, which are likely sensory cells (Figure 5H-J). In late stage larvae, expression is limited to a ring in the ectoderm of the hindgut (Figure $5 \mathrm{~L}$ ). In some cases we also observed weak expression in the PGZ (not shown), which we interpret to be residual transcript from expression during earlier stages.

\section{Ct-Pax3/7 expression}

Ct-Pax3/7 transcripts are detected at all developmental stages examined (Stage 3 to 9 and in juveniles) in two ventrolateral ectodermal bands oriented along the anterior-posterior axis. Following gastrulation at Stage 3, Ct$\mathrm{Pax} 3 / 7$ is expressed in two small lateral domains in the posterior half of the embryo (Figure 6A). Slightly later, this expression expands anteriorly to form two bands of ectodermal expression (Figure 6B,C). This expression appears prior to the formation of the larval segments, prototroch, telotroch and the ventral nerve cord. The lateral bands of $\mathrm{Ct}$-Pax3/7 expression continue to expand anteriorly and by Stage 4 they extend from the level of the mouth to the telotroch (Figure 6E,F). The longitudinal bands of $\mathrm{Ct}$-Pax3/7 expression are the only detectable expression domains at every stage examined, including immediately prior to and during the appearance of the first segments (Figure $6 \mathrm{C}-\mathrm{H}$ ). The relative intensity of expression varies somewhat along the length of the band (Figure 6G). At Stage 6, expression in anterior segments decreases (Figure 6J), and gradually decreases from anterior to posterior. The posterior boundary of expression is stable from Stages 5 to 9; it abuts, but does not include the telotroch. Between Stage 4 and 6 , the distance between the two bands of expression decreases (compare Figure 6G and $6 \mathrm{~J}$ ), reflecting the movement of the two sides of the ventral nerve cord towards the ventral midline. By late Stage 7, Ct-Pax3/7 expression is limited to a small ventrolateral domain of ectoderm in the PGZ (Figure 6K). This expression is positioned immediately lateral to the ventral ganglia (Figure 6L), and persists through Stage 8 (Figure 6M). The Ct-Pax3/7 transcript is no longer detectable in Stage 9 larvae. In post-metamorphic juveniles, $\mathrm{Ct}$-Pax3/ 7 expression is limited to a small ventrolateral domain of ectoderm that abuts the lateral edge of the ventral ganglia in the nascent segment and also extends into the growth zone (Figure 6N,O,P). Thus, in juveniles, $\mathrm{Ct}$ Pax3/7 is expressed in a restricted area of ectoderm in the PGZ.

\section{Expression of eve genes}

Although the two C. teleta eve genes have close to $100 \%$ amino acid identity within their ORFs (see previous section), each gene has a unique expression pattern. Both genes are detected at all developmental stages examined in multiple tissues and multiple germ layers (Stages 3 to 9). However, neither Ct-eve1 nor Ct-eve2 is detected in ectodermal or mesodermal stripes in the mid-body, whether preceding or following the appearance of segments (Figures 7 and 8). Ct-eve1 is broadly expressed during cleavage stages (data not shown); here, we focus on spatial characterization of the Ct-eve1 transcript from the end of gastrulation (Stage 3) through metamorphosis (Stage 9). During gastrulation, when a blastopore is present (Stage 3b), Ct-eve1 is detected in two domains: in the mesodermal precursor cells (Figure 7A) and the dorsal side of the embryo, in the presumptive brain (Figure 7B). Soon after, Ct-eve1 is transiently expressed in the endoderm (Figure 7C). In addition, expression persists in the nascent mesodermal bands and in the forming brain (Figure 7C). During Stage 3, Ct-eve1 also is expressed in a fourth domain in a posterior ring of ectodermal cells that extend to the dorsal surface of the embryo (Figure 7D). Expression associated with the presumptive foregut and hindgut appears at Stage 4 (Figure 7E). The Ct-eve1 transcript is broadly expressed throughout the mid-body mesoderm as it expands circumferentially through Stage 5 and 6 (Figure 7F,G,I). Expression also persists in the mesodermal precursor cells, which at this stage are positioned beneath the telotroch (Figure 4C and 7I, large arrows). During this time, expression in the brain, foregut and hindgut persists from previous stages (Figure 7G,H,I). In late larval stages, there is expression in the posterior mid-body mesoderm, which gradually becomes restricted to the PGZ by Stage 9 (Figure $7 \mathrm{~J}, \mathrm{~K}, \mathrm{~L}$ ), and expression in other domains is either very weak or not detectable. In juvenile stages, there is a continuous domain of expression that includes the ectoderm and mesoderm of the PGZ and the nascent segments (Figure $9 \mathrm{~A}, \mathrm{C})$. Ct-eve1 is also expressed in posterior ganglia of juveniles.

Ct-eve2 has distinct expression domains from Ct-eve1, although there are clear overlapping areas of expression. When the blastopore is present (Stage $3 \mathrm{~b}$ ), Ct-eve2 is detected in the mesodermal precursor cells (Figure 8A) and on the posterior face of the blastopore at the ventral midline (Figure 8B). Later during Stage 3, there is continued expression in the mesodermal precursor cells, and novel expression in the presumptive hindgut (Figure $8 \mathrm{C}$ ) and in a ring of ectodermal cells near the posterior end of the embryo (Figure 8D). At Stages 4 and 5, after the telotroch has formed, it is apparent that these posterior ectodermal cells are immediately anterior to the telotroch in the position of the presumptive PGZ (not shown). Ct-eve2 expression in the mesodermal precursor cells and hindgut persists (Figure 8E,G). At Stage 5, Cteve 2 also appears in several additional ectodermal domains, including weak expression in the foregut and mid-body ectoderm (between the prototroch and telotroch). The most prominent expression is in the forming 


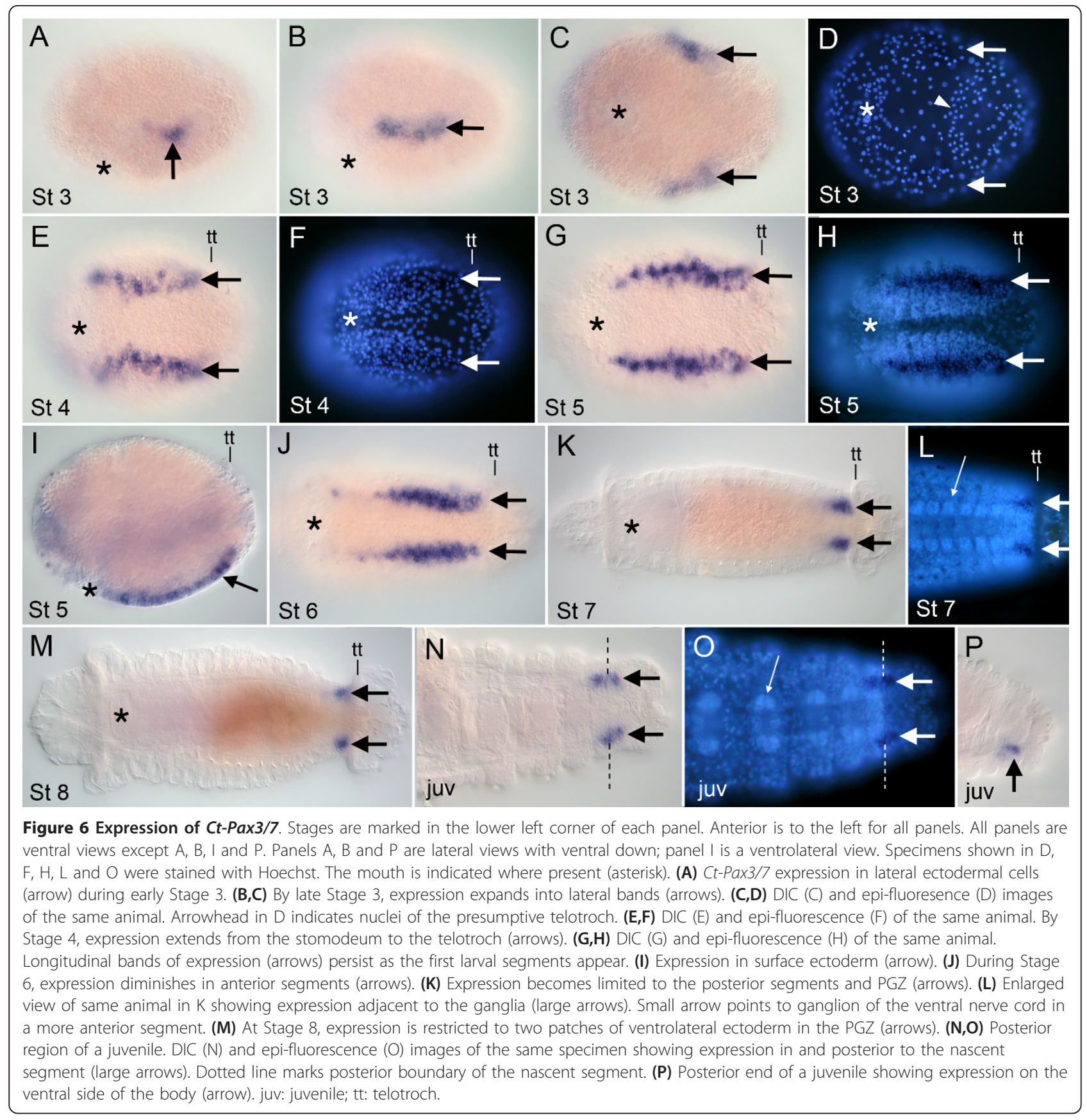

ventral nerve cord (Figure 8F), which persists as the left and right sides fuse at the ventral midline at Stage 6 (Figure $8 \mathrm{H}$ ). In the mid-body, there is expression in both the ectoderm and mesoderm at Stage 6. The mesodermal expression is particularly pronounced in the PGZ and in the mesodermal precursor cells (Figure 8I). In the ectoderm, small clusters of cells express Ct-eve2 more strongly relative to surrounding cells (Figure $8 \mathrm{H}$ arrowheads), which become refined to three columns of discrete, segmentally iterated cell clusters on each side of the animal by Stage 7 (Figure 8 J,K,L). The three columns are in the following positions: between the ventral nerve cord and the neuropodial chaetae, between the neuro- and notopodial chaetae, and dorsal to the notopodial chaetae (Figure 8J). The most dorsal column is the first to express $\mathrm{Ct}$-eve2. These three columns of $\mathrm{Ct}$ eve2-expressing cell clusters span the ectoderm epithelia and have a very similar expression pattern to elav [40] and synaptotagmin (Meyer and Seaver, unpublished data), conserved markers of neuronal differentiation and 


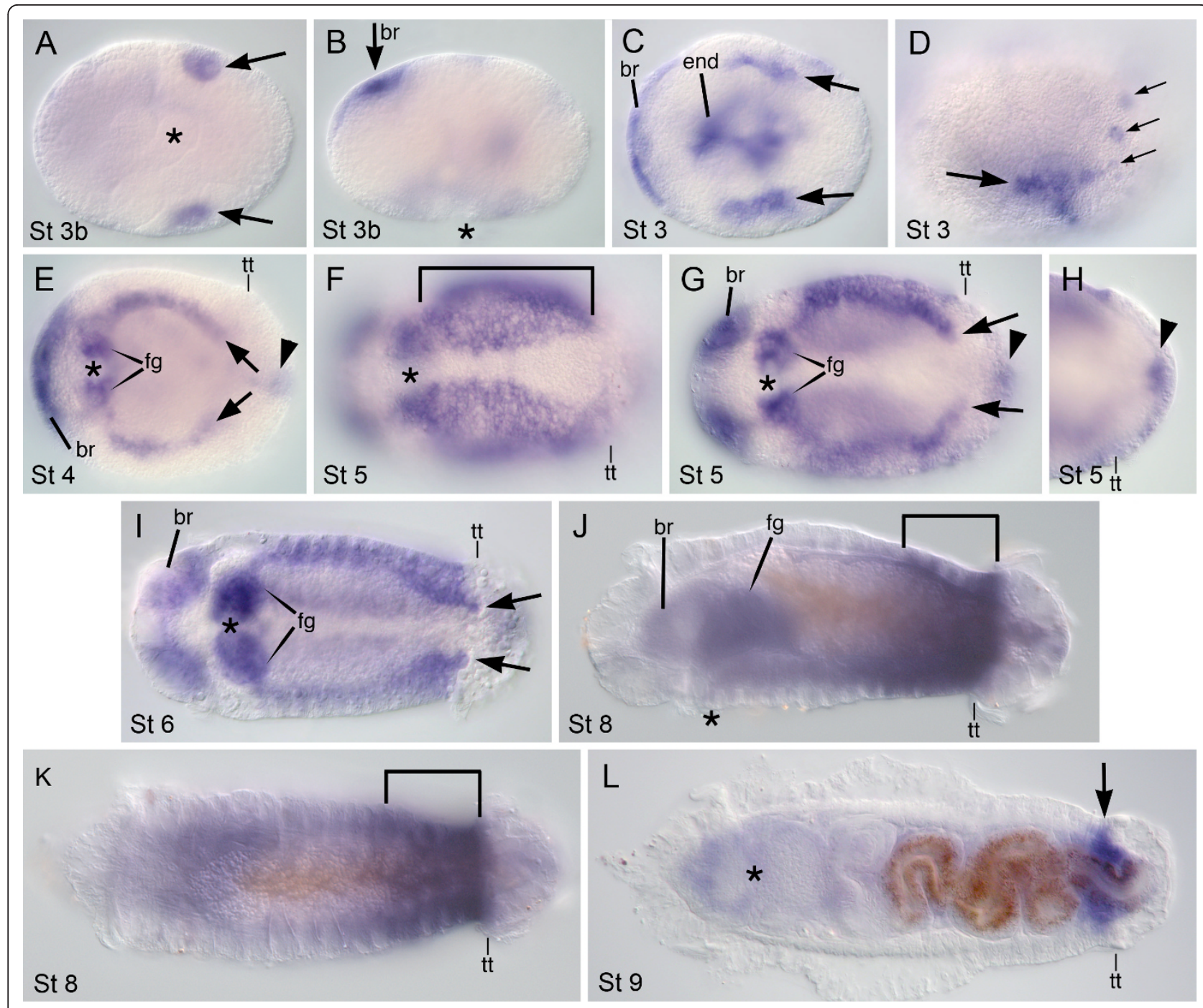

Figure 7 Expression of Ct-eve1. Stages are marked in the lower left of each panel and anterior is to the left for all panels. Except for B, D, J (lateral views with ventral down) and $\mathrm{K}$ (dorsal view), all panels are ventral views. An asterisk marks the blastopore in $A$ and $B$ and the mouth in all other panels. $(\mathbf{A}, \mathbf{B})$ Ventral $(A)$ and lateral $(B)$ views of the same animal. At the end of gastrulation, $C t$-evel is expressed in mesodermal precursor cells (arrows in A) and the presumptive brain (br). (C,D) Expression in the presumptive brain (br), mesodermal bands (large arrows), endoderm (end) and ectodermal cells positioned immediately anterior to the telotroch (small arrows). Panel C is a composite of multiple focal planes. (E) Expression is in the brain (br), presumptive foregut (fg), mesodermal bands (large arrows) and hindgut (arrowhead). (F-H) Expression is throughout the mid-body mesoderm as it expands circumferentially (bracket in F, arrows in G) and there is continued expression in the brain (br), foregut (fg) and hindgut (arrowhead). F-H are different focal planes of the same animal. (I) Expression during Stage 6 includes the brain (br), foregut (fg), mid-body mesoderm and mesodermal precursor cells (arrows). (J,K) By Stage 8, the most prominent expression is in the posterior mid-body (bracket), with weaker expression in the brain, foregut and hindgut. (L) In late larval stages, expression is largely restricted to the PGZ (arrow). br: brain; end: endoderm; fg: foregut; St 3b: Stage 3 blastopore; tt: telotroch.

of differentiated neurons, respectively. These columns of cell clusters are likely peripheral sensory structures. In addition, at Stages 6 and 7, there is ectodermal expression in a single line of cells across the dorsal surface of some larvae in the PGZ (Figure 8L). This expression domain is not visible at later stages. By Stage 8 , there is only weak expression in the columns of ectodermal cell clusters (not shown) and they are undetectable by Stage 9. Expression persists in the hindgut and PGZ into
Stage 8 (Figure $8 \mathrm{M}, \mathrm{O}, \mathrm{P}$ ), and at this stage it is clear from the subsurface position of the hindgut expression that $\mathrm{Ct}$-eve 2 is localized to the rectum. A new expression domain appears at Stage 8 in mesodermal cells along the dorsal midline in the posterior mid-body (Figure $8 \mathrm{M}, \mathrm{N}, \mathrm{O})$. These Ct-eve2-expressing cells can be seen extending from the dorsal midline along the anterior edge of segments (Figure $8 \mathrm{~N}$ ). It is unknown to what structures these cells contribute. At Stage 9, 


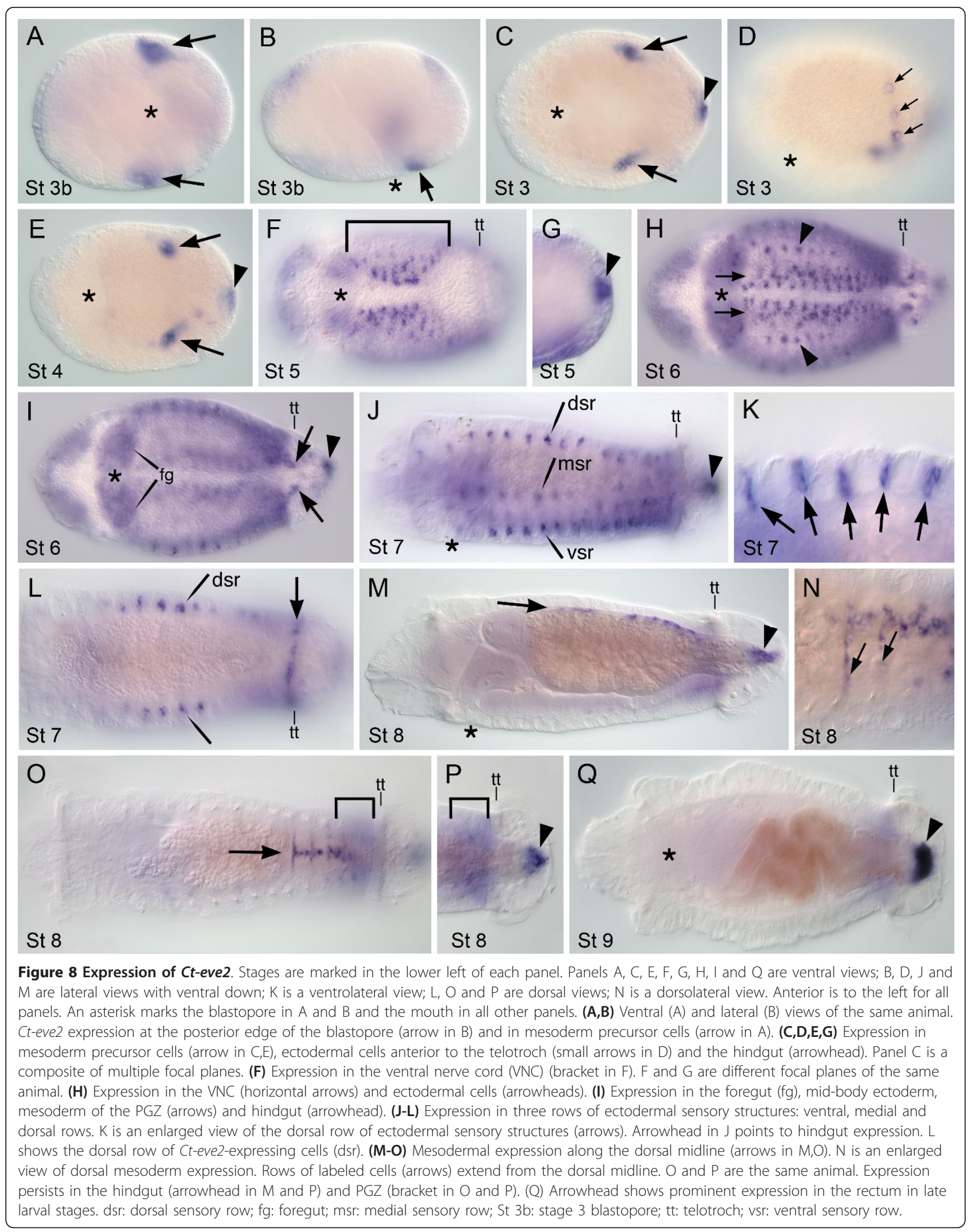


expression is limited to a small domain in the rectum (Figure $8 \mathrm{Q}$ ) and weak expression in the PGZ (Figure $8 \mathrm{Q}$ ) and dorsal midline (not shown). In juveniles, $C t$ eve 2 is expressed in the PGZ, hindgut and weakly in posterior ganglia (Figure 9B,D).

In summary, Ct-eve1 and Ct-eve 2 have unique spatiotemporal expression patterns, although they are both expressed in mesodermal precursor cells, foregut and hindgut, and the PGZ. Prior to the generation of the mesodermal bands, Ct-eve1 and Ct-eve 2 are similarly expressed in the mesodermal precursor cells (compare Figures $7 \mathrm{~A}$ and $8 \mathrm{~A}$ ). Once the mesodermal bands appear, however, Ct-eve1 is expressed in both mesodermal precursor cells and their descendant bands, whereas Ct-eve2 is restricted to the mesodermal precursor cells and is not expressed in their progeny. The temporal dynamics of hindgut expression between the two genes is distinct. Ct-eve2 is continuously expressed in the hindgut from Stage 3 through metamorphosis into juvenile stages (Figures 8 and 9). In contrast, Ct-eve1 expression is transient in the hindgut, is initially detected a day later than for Ct-eve 2 at Stage 4, and is not detectable in late stage larvae (compare Figures $7 \mathrm{~L}$ and $8 \mathrm{Q}$ ). Another difference between $C t$-eve 1 and $C t$-eve2 expression is in the mid-body, where Ct-eve 2 is largely expressed in ectodermal derivatives such as the ventral nerve cord and peripheral sensory structures. This differs dramatically from Ct-eve1, which has broad expression throughout the mid-body mesoderm. The unique expression patterns for $C t$-eve 1 and $C t$-eve 2 indicates a divergence of regulatory elements between these two genes.

\section{Discussion}

\section{Runt, Pax3/7 and eve genes in C. teleta}

The C. teleta genome has clear orthologs of the D. melanogaster pair-rule segmentation genes runt, paired (Pax3/ $7)$ and eve. Two eve genes are present in the C. teleta genome and, based on amino acid sequence similarity and genomic linkage, they appear to be either the result of a recent duplication or concerted evolution, possibly due to gene conversion event(s) $[55,56]$. Gene conversion has previously been proposed to occur between the two $D l x$ genes in the C. teleta genome [57]. Both Ct-Pax3/7 and Ct-runt lack a Groucho-binding domain, which is conserved in orthologs of these genes from other species. However, the Pax3/7A ortholog in the leech Helobdella sp. [58] and the sea squirt Ciona intestinalis [59], and the D. melanogaster paired gene [60] also lack this motif, as does the predicted limpet Lottia gigantea runt homolog. The C. teleta genome contains a groucho gene (scaffold_692 1858), and other C. teleta genes contain the groucho repression motif, including the hes genes [29]. These features confirm that the groucho repression motif is present and likely utilized in the context of transcriptional
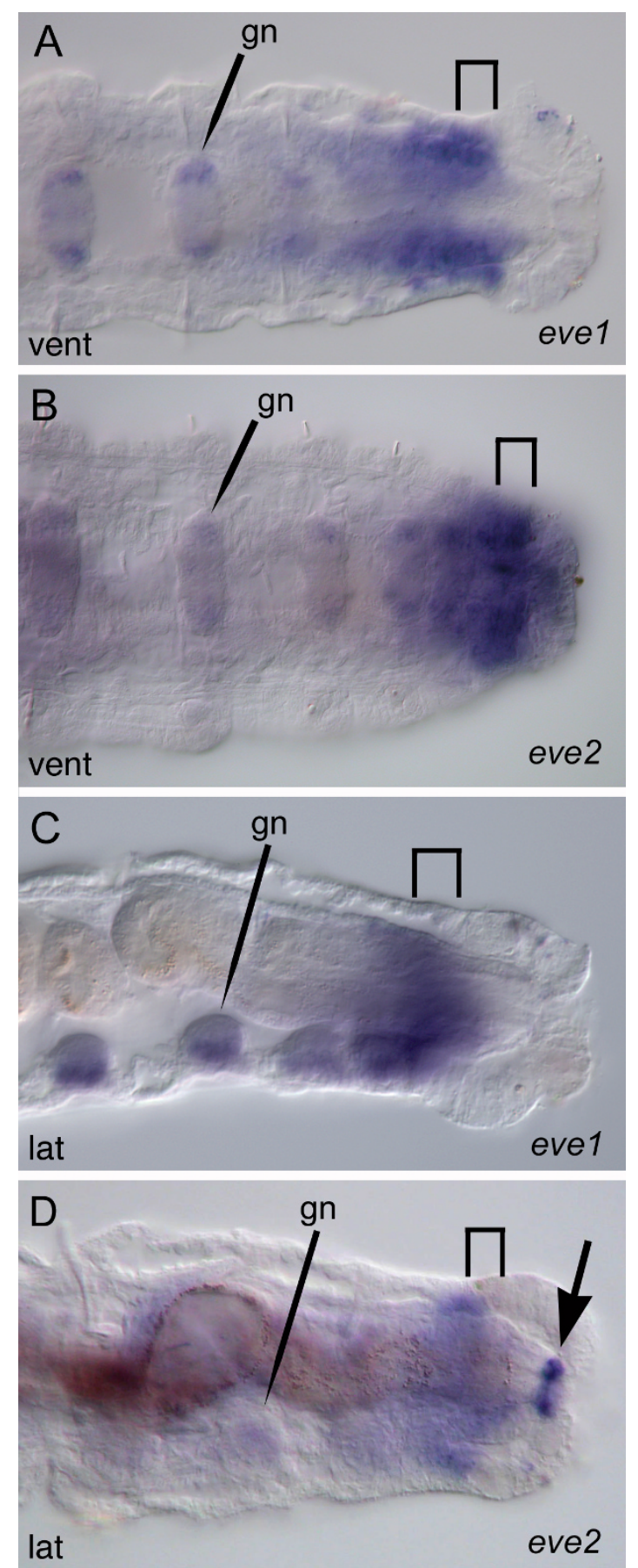

Figure 9 Ct-eve 1 and $C$-eve 2 expression in the posterior growth zone of juveniles. All panels show the posterior end of juveniles. $A$ and $B$ are ventral (vent) views and $C$ and $D$ are lateral (lat) views with ventral down. A bracket denotes the PGZ. (A) Ctevel is expressed in the ectoderm and mesoderm of the PGZ and in the ganglia of the ventral nerve cord (VNC). (B) Ct-eve2 is expressed in the PGZ and weakly in the ganglia of the VNC. (C) Ctevel expression in the PGZ is more prominent on the ventral side than on the dorsal side (bracket). (D) In addition to PGZ expression in the ectoderm and mesoderm (bracket), Ct-eve2 is expressed in the anus (arrow). gn: ganglion; lat: lateral; vent: ventral. 
co-repression in C. teleta. Although we do not know the functional consequences of the lack of a groucho domain on the C. teleta runt and paired homologs, the lack of a groucho domain in $D$. melanogaster paired suggests that this domain is not necessary for a segmentation function.

\section{Relationship of Ct-runt, Ct-Pax3/7, Ct-eve1 and Ct-eve2 expression to segment formation}

If annelids and arthropods shared a common segmented ancestor, the expectation is that pair-rule gene orthologs would be expressed in transverse stripes with a consistent relationship to segment boundaries in C. teleta, with either a pair-rule or segmental periodicity. However, none of the genes we examined, Ct-runt, Ct-Pax3/7, Cteve1 or $C t$-eve2, have a spatio-temporal expression pattern consistent with a possible role in segment formation. When the first larval segments are forming, Ct-runt expression is limited to a subset of cells in the brain and there is no expression in the mid-body where new segments form. Although Ct-Pax3/7 is expressed in the mid-body prior to overt segment formation in larvae, its spatial pattern does not prefigure segments, either in a pair-rule or segmental pattern. Both Ct-eve genes are expressed in the mid-body as larval segments begin to form. At these stages, the Ct-eve1 transcript is ubiquitously expressed in the mid-body mesoderm and lacks spatially restricted positional expression expected for a role segment boundary formation. Ct-eve2 expression has more restricted expression in the mid-body, limited to mesodermal precursor cells and the ventral nerve cord, and also lacks pair-rule or segmental stripes of expression in the ectoderm or mesoderm. At late larval stages and during juvenile growth when segments form from the PGZ, all four genes are expressed in the PGZ. However, none of them have patterns that prefigure segments, such as circumferential banded expression or expression limited to either the anterior or posterior region of the growth zone. Ct-Pax3/7 and Ct-runt have restricted expression around the circumference of the PGZ, in a small ventrolateral domain and ventrolateral domain of the ectoderm, respectively. Both $C t$-eve genes have broad posterior expression domains that include the PGZ and nascent segments in late larval stages and juveniles, but do not resolve into more restricted patterns. In some animals that form segments sequentially, such as in vertebrates, centipedes and spiders, segment formation is preceded by waves of dynamic gene expression [61-63]. Of the genes examined here, we did not observe dynamic expression in the PGZ, since we did not detect multiple patterns in the PGZ for a gene in similarly staged larvae.

We previously examined expression of orthologs of the pair-rule genes odd-paired and hairy in C. teleta [29,30]. During early stages of segment formation, Ct-zic, the C. teleta ortholog of odd-paired, is expressed along the lateral edge of the ventral neural ectoderm, and in segmentally iterated domains of mesoderm expression in the mid-body soon after segments form. However, there are no stripes of expression in either the ectoderm or mesoderm. One of the hairy orthologs, CapI-hes1, is expressed in the mesoderm of presumptive larval segments in a segmentally iterated pattern, although it is very distinct from arthropod patterns of hairy expression $[7,64,65]$. Of the pair-rule orthologs for which we have examined the expression pattern in C. teleta, CapI-hes 1 is the most promising candidate to function in segmentation [29]. Ct-Pax3/7, Ct-runt, Ct-eve1, Ct-eve2, CapI-hes 2 and $C t$-zic all have very distinct expression patterns, all of which indicate that these genes are not likely to be involved in segmentation. Perhaps a subset of these genes are post-transcriptionally regulated and play a role in establishing segmental boundaries; however, functional studies will be necessary to obtain a definitive confirmation of the role of these pair-rule homologs in C. teleta.

\section{Comparison of runt, Pax3/7 and eve expression among species}

Expression of the pair-rule genes $\mathrm{Pax} 3 / 7$ and eve have been characterized in a few other annelids, but expression patterns for runt have not been reported for any other lophotrochozoan. Runt orthologs appear to have a conserved role in D. melanogaster [66] and in Danio rerio [67], chick [68] and mammalian neural development (reviewed in [69]), and expression of Ct-runt in the brain and ventral nerve cord of $C$. teleta extends this conservation. For $\mathrm{Pax} 3 / 7$ orthologs, expression has been examined in two other annelids. Expression of $\mathrm{Pax3} / 7$ in $P$. dumerilii is somewhat similar to the pattern in C. teleta [70]. Both Pdu-pax3/7 and Ct-Pax3/7 are expressed in bilaterally symmetric longitudinal bands in the ventral trunk ectoderm, adjacent to the ventral nerve cord. In $P$. dumerilii, each band of expression is much broader than in C. teleta, possibly reflecting differences in the size of the neurogenic field at these stages. Helobdella sp. Austin has two Pax3/7 orthologs. The expression pattern has been reported for Hro-Pax $3 / 7 A$, and it is expressed in the mesodermal bands and later in mesodermal derivatives [58]. This is in contrast to the longitudinal bands of Pax3/7 expression in the ventral ectoderm in C. teleta and $P$. dumerilii. A better understanding of the extent to which these genes have conserved expression domains in annelids will emerge as data from additional species are reported. Outside of annelids, $\mathrm{Pax} 3 / 7$ orthologs function in neural specification in the ventral nerve cord of $D$. melanogaster and the vertebrate neural tube. However, their expression patterns are quite distinct in that there are transverse bands of expression in D. melanogaster and longitudinal bands of expression in vertebrates $[71,72]$. 
Comparison of eve expression patterns across bilaterian animals reveals several common expression domains. In annelids, eve is expressed in the growth zone during posterior growth of C. teleta and H. robusta, and in the PGZ during regeneration of $P$. dumerilii [73,74]. However, in P. dumerilii, expression in the PGZ is ectodermal, whereas in $H$. robusta and C. teleta it is both ectodermal and mesodermal. Furthermore, there is prominent expression in the mesodermal bands of $C$. teleta and $H$. robusta, but not in P. dumerilii. Additionally, eve is expressed in the posterior gut of both $C$. teleta and $P$. dumerilii. Outside of annelids, posterior expression of eve has been reported in Danio rerio [75], Xenopus laevis [76], B. floridae [46], nematodes [77], and in a range of arthropods [10,15,17,78-80]. Eve also appears to have an important and widespread role in neural specification or neurogenesis in bilaterians, including in insects (D. melanogaster [48,81], Schistocerca americana [10]), Caenorhabditis elegans [82], B. floridae [46], $X$. laevis [83] and Mus musculus [84]. In annelids, eve is expressed in the developing ventral nerve cord of C. teleta, $P$. dumerilii and $H$. robusta, and a role in neural development appears to be the most conserved function of eve. Although less well characterized, eve has also been implicated in mesodermal patterning in Danio rerio [85] and D. melanogaster [48]. The expression of eve in pericardial progenitors along the dorsal midline in $D$. melanogaster and in mesodermal cells along the dorsal midline of C. teleta may indicate a shared role in protostome cardiovascular development. In summary, eve genes show conserved roles across bilaterians in multiple domains, indicating conservation of several developmental roles, and the C. teleta eve gene expression patterns appear to show conservation with several of these functions. This broad conservation for eve is in contrast with its function in arthropod segmentation, which appears to be more of a clade-specific role.

\section{Conclusions}

To the best of our knowledge, no orthologs of D. melanogaster pair-rule genes show striped patterns of ectodermal expression in annelids, either in a segmental or pairrule-like pattern. Vertebrate orthologs of runt, Pax3/7 and eve do not have a role in somitogenesis. Furthermore, a pair-rule-like expression pattern has not been reported for any gene in annelids. In the polychaete $P$. dumerilii, a number of genes are reported to be expressed in segmental ectodermal stripes during larval segment formation, adult segment generation and/or during regeneration. These include members of the $N K$ gene family, specifically $N K 4, L b x$ and $M s x$ orthologs [86], the wnt genes Wnt1, Wnt4, Wnt5, Wnt10, Wnt11, Wnt16 [24,87], hh [88] and en [24]. However, with the exception of en, $h h, W n t 1$ and $W n t 5$, these genes do not function in either arthropod or vertebrate segmentation, and thus are unlikely to be present in an ancestral bilaterian segmentation cascade. Furthermore, there are no functional data implicating any of these genes in the initial formation of segmental boundaries in annelids. Thus, there are likely fundamental differences in how segments form in annelids and arthropods, with no evidence of pair-rule patterning as part of the annelid segmentation program.

\section{Additional material}

Additional file 1: Table S1. List of animal species, gene abbreviations
and NCBI accession numbers used for amino acid sequence alignments.
Additional file 2: Figure S1. Ct-Runt groups with Runt protein
sequences from other animal taxa. The tree shown is a Bayesian
consensus tree. Posterior probability support is indicated above the
nodes, and maximum likelihood bootstrap support values are indicated
below the nodes where the two tree topologies agree. Abbreviations are
as in the Pax family tree (Figure 2), with the following additional taxa:
Amq: Amphimedon queenslandica; Bm: Bombyx mori; Cs-: Cupiennius salei;
Fr: Takifugu rubripes; Nv: Nematostella vectensis; Sk: Saccoglossus
kowalevskii; Sm: Schistosoma mansoni; Sp: Strongylocentrotus purpuratus;
Tc: Tribolium castaneum.
Additional file 3: Figure S2. The two C. teleta Eve proteins group
together, within a clade that contains Eve sequences from other
animals. The tree shown is a Bayesian consensus tree, with posterior
probability support placed above the nodes; maximum likelihood
bootstrap support values are placed below the node where the tree
topologies agree. Abbreviations are as in the Pax family tree (Figure 2),
with the following additional taxa: Aaeg: Aedes aegyptii; Ce:
Caenorhabditis elegans; Hro-: Helobdella robusta; lo: llyanassa obsoleta; Lg:
Lottia gigantea; Stm-: Strigamia maritime; Ttr-: Theromyzon trizonare.

\section{Acknowledgements}

We thank Alexa Bely for the forward eve primer, Maja Makagon for cloning the initial fragment of eve from C. teleta, Lori Kaneshige for technical assistance with cloning and in situ hybridization, Danielle de Jong for help with genomic analysis of the eve genes, and Greg Davis and Nipam Patel for the Prby degenerate primer sequences prior to publication. We are grateful to members of Kewalo Marine Laboratory for discussions and comments on the preparation of this manuscript. The use of the PBRC cluster for RAxML maximum likelihood analyses comes from The University of Hawaii RCMI Program (G12RR003061). This work was supported by the National Science Foundation (IOB00-94925 and EAR-0342392 to ECS).

\section{Author details}

${ }^{1}$ Kewalo Marine Laboratory, Pacific Biosciences Research Center, University of Hawaii, 41 Ahui Street, Honolulu, HI, USA. ${ }^{2}$ Sars International Centre for Marine Molecular Biology, Bergen, Norway. ${ }^{3}$ Biology Department, Clark University, Worcester, MA, USA.

\section{Authors' contributions}

ECS designed the study, performed in situ hybridization experiments, did the critical analysis of the data, imaged specimens, prepared figures and wrote the manuscript. GR contributed to gene cloning, generation of riboprobes, in situ hybridization experiments and carried out gene orthology analyses. EY carried out gene orthology analyses and contributed to figure preparation and writing of the manuscript. NM carried out staining, confocal microscopy and contributed to figure preparation. All authors contributed to editing of the manuscript and all authors read and approved the final manuscript.

\section{Competing interests}

The authors declare that they have no competing interests. 
Received: 28 November 2011 Accepted: 18 April 2012

Published: 18 April 2012

\section{References}

1. Blair SS: Segmentation in animals. Curr Biol 2008, 18:R991-995.

2. Chipman A: Parallel evolution of segmentation by co-option of ancestral gene regulatory networks. Bioessays 2010, 32:60-70.

3. Peel A, Akam M: Evolution of segmentation: rolling back the clock. Curr Biol 2003, 13:R708-710.

4. Seaver EC: Segmentation: mono- or polyphyletic. Int J Dev Biol 2003, 47:583-595.

5. Hejnol A, Obst M, Stamatakis A, Ott M, Rouse GW, Edgecombe GD, Martinez P, Baguñà J, Bailly X, Jondelius U, Wiens M, Müller WE, Seaver E, Wheeler WC, Martindale MQ, Giribet G, Dunn CW: Rooting the bilaterian tree with scalable phylogenomic and supercomputing tools. Proc Biol Sci 2009, 276:4261-4270

6. Pick KS, Philippe H, Schreiber F, Erpenbeck D, Jackson DJ, Wrede P, Wiens M, Alie A, Morgenstern B, Manuel M, Worheide G: Improved phylogenomic taxon sampling noticeably affects nonbilaterian relationships. Mol Biol Evol 2010, 27:1983-1987.

7. Damen WGM: Evolutionary conservation and divergence of the segmentation process in arthropods. Dev Dyn 2007, 236:1379-1391.

8. Nüsslein-Volhard C, Wieschaus E: Mutations affecting segment number and polarity in Drosophila. Nature 1980, 287:795-801.

9. Davis GK, Patel NH: Short, long, and beyond: molecular and embryological approaches to insect segmentation. Annu Rev Entomol 2002, 47:669-699.

10. Patel NH, Ball EE, Goodman CS: The changing role of even-skipped during the evolution of insect pattern formation. Nature 1992, 357:339-342.

11. Davis GK, D'Alessio JA, Patel NH: Pax3/7 genes reveal conservation and divergence in the arthropod segmentation hierarchy. Dev Biol 2005, 285:169-184.

12. Schoppmeier M, Damen WG: Expression of Pax group III genes suggests a single-segmental periodicity for opisthosomal segment patterning in the spider Cupiennius salei. Evol Dev 2005, 7:160-169.

13. Dearden P, Donly C, Grbic M: Expression of pair-rule gene homologues in a chelicerate: early patterning of the two-spotted spider mite Tetranychus urticae. Development 2002, 129:5461-5472.

14. Janssen R, Budd GE, Prpic N-M, Damen WG: Expression of myriapod pair rule gene orthologs. EvoDevo 2011, 2(1):5.

15. Hughes $\mathrm{CL}$, Kaufman TC: Exploring myriapod segmentation: the expression patterns of even-skipped, engrailed, and wingless in a centipede. Dev Biol 2002, 247:47-61.

16. Damen WGM, Janssen R, Prpic N-M: Pair rule gene orthologues in spider segmentation. Evol Dev 2005, 7:618-628.

17. Liu PZ, Kaufman TC: Even-skipped is not a pair-rule gene but has segmental and gap-like functions in Oncopeltus fasciatus, an intermediate germband insect. Development 2005, 132:2081-2092.

18. Davis GK, Patel NH: Playing by pair-rules? Bioessays 2003, 25:425-429.

19. Gutjahr T, Frei E, Noll M: Complex regulation of early paired expression: initial activation by gap genes and pattern modulation by pair-rule genes. Development 1993, 117:609-623.

20. Ingham PW, Baker NE, Martinez-Arias A: Regulation of segment polarity genes in the Drosophila blastoderm by fushi tarazu and even skipped. Nature 1988, 331:73-75.

21. Damen WG: Parasegmental organization of the spider embryo implies that the parasegment is an evolutionary conserved entity in arthropod embryogenesis. Development 2002, 129:1239-1250.

22. Patel NH, Kornberg TB, Goodman CS: Expression of engrailed during segmentation in grasshopper and crayfish. Development 1989, 107:201-212

23. Scholtz G, Patel NH, Dohle W: Serially homologous engrailed stripes are generated via different cell lineages in the germ band of amphipod crustaceans (Malacostraca, Peracarida). Intl J Dev Biol 1994, 38:471-478.

24. Prud'homme B, de Rosa R, Arendt D, Julien JF, Pajaziti R, Dorresteijn A, Adoutte A, Wittbrodt J, Balavoine G: Arthropod-like expression patterns of engrailed and wingless in the annelid Platynereis dumerilii suggest a role in segment formation. Curr Biol 2003, 13:1876-1881.

25. Seaver EC, Paulson DA, Irvine SQ, Martindale MQ: The spatial and temporal expression of $\mathrm{Ch}$-en, the engrailed gene in the polychaete Chaetopterus, does not support a role in body axis segmentation. Dev Biol 2001 236:195-209

26. Seaver EC, Kaneshige LM: Expression of 'segmentation' genes during larval and juvenile development in the polychaetes Capitella sp. I and $\mathrm{H}$. elegans. Dev Biol 2006, 289:179-194.

27. Blake JA, Grassle JP, Eckelbarger KJ: Capitella teleta, a new species designation for the opportunistic and experimental Capitella sp. I, with a review of the literature for confirmed records. Zoosymposia 2009, 2:25-53.

28. Lans D, Wedeen CJ, Weisblat DA: Cell lineage analysis of the expression of an engrailed homolog in leech embryos. Development 1993, 117:857-871.

29. Thamm K, Seaver EC: Notch signaling during larval and juvenile development in the polychaete annelid Capitella sp. I Dev Biol 2008 320:304-318.

30. Layden MJ, Meyer NP, Pang K, Seaver EC, Martindale MQ: Expression and phylogenetic analysis of the zic gene family in the evolution and development of metazoans. EvoDevo 2010, 1(1):12.

31. Grassle J, Grassle JF: Sibling species in the marine pollution indicator Capitella (polychaeta). Science 1976, 192:567-569.

32. Seaver EC, Thamm K, Hill SD: Growth patterns during segmentation in the two polychaete annelids, Capitella sp. I and Hydroides elegans: comparisons at distinct life history stages. Evol Dev 2005, 7:312-326.

33. Damen WGM, Weller M, Tautz D: Expression patterns of hairy, evenskipped, and runt in the spider Cupiennius salei imply that these genes were segmentation genes in a basal arthropod. PNAS 2000, 97:4515-4519.

34. Davis GK, Jaramillo CA, Patel NH: Pax group III genes and the evolution of insect pair-rule patterning. Development 2001, 128:3445-3458.

35. Capitella teleta v1.0. [http://genome.jgi-psf.org/Lotgi1/Lotgi1.home.html].

36. Abascal F, Zardoya R, Posada D: ProtTest: selection of best-fit models of protein evolution. Bioinformatics 2005, 21:2104-2105.

37. Huelsenbeck JP, Ronquist F: Bayesian analysis of molecular evolution using MrBayes. In Statistical methods in molecular evolution. Edited by: Nielsen R. New York: Springer; 2005:504.

38. Stamatakis A: RAxML-VI-HPC: maximum likelihood-based phylogenetic analyses with thousands of taxa and mixed models. Bioinformatics 2006 22:2688-2690.

39. FigTree v1.3.1. [http://tree.bio.ed.ac.uk/software/figtree/].

40. Meyer NP, Seaver EC: Neurogenesis in an annelid: characterization of neural progenitors in the polychaete Capitella sp. I Dev Biol 2009, 335:237-252

41. Aronson BD, Fisher AL, Blechman K, Caudy M, Gergen JP: Grouchodependent and -independent repression activities of Runt domain proteins. Mol Cell Biol 1997, 17:5581-5587.

42. Lottia gigantea v1.0. [http://genome.jgi-psf.org/Lotgi1/Lotgi1.info.html].

43. Balczarek KA, Lai ZC, Kumar S: Evolution of functional diversification of the paired box (Pax) DNA-binding domains. Mol Biol Evol 1997, 14:829-842.

44. Matus DQ, Pang K, Daly M, Martindale MQ: Expression of Pax gene family members in the anthozoan cnidarian, Nematostella vectensis. Evol Dev 2007, 9:25-38.

45. Schmerer M, Savage RM, Shankland M: Pax $\beta$ : a novel family of lophotrochozoan Pax genes. Evol Dev 2009, 11:689-696.

46. Ferrier DE, Minguillon C, Cebrian C, Garcia-Fernandez J: Amphioxus Evx genes: implications for the evolution of the midbrain-hindbrain boundary and the chordate tailbud. Dev Biol 2001, 237:270-281.

47. Brown SJ, Parrish JK, Beeman RW, Denell RE: Molecular characterization and embryonic expression of the even-skipped ortholog of Tribolium castaneum. Mech Dev 1997, 61:165-173.

48. Frasch M, Hoey T, Rushlow C, Doyle H, Levine M: Characterization and localization of the even-skipped protein of Drosophila. EMBO J 1987, 6:749-759

49. Fröbius AC, Seaver EC: Genomic organization and expression demonstrate spatial and temporal Hox gene colinearity in the lophotrochozoan Capitella sp. I. PLOS ONE 2008, 3:e4004.

50. Hui J, McDougall C, Monteiro A, Holland P, Arendt D, Balavoine G, Ferrier D: Extensive chordate and annelid macrosynteny reveals ancestral homeobox gene organization. Mol Biol Evol 2011, 29:7-165.

51. Holland LZ, Albalat R, Azumi K, Benito-Gutierrez E, Blow MJ, BronnerFraser M, Brunet F, Butts T, Candiani S, Dishaw LJ, Ferrier DE, GarciaFernàndez J, Gibson-Brown JJ, Gissi C, Godzik A, Hallböök F, Hirose D, Hosomichi K, Ikuta T, Inoko H, Kasahara M, Kasamatsu J, Kawashima T, 
Kimura A, Kobayashi M, Kozmik Z, Kubokawa K, Laudet V, Litman GW, McHardy AC, Meulemans D, Nonaka M, Olinski RP, Pancer Z, Pennacchio LA, Pestarino M, Rast JP, Rigoutsos I, Robinson-Rechavi M, Roch G, Saiga H, Sasakura $Y$, Satake M, Satou Y, Schubert M, Sherwood N, Shiina T, Takatori N, Tello J, Vopalensky P, Wada S, Xu A, Ye Y, Yoshida K, Yoshizaki F, Yu JK, Zhang Q, Zmasek CM, de Jong PJ, Osoegawa K, Putnam NH, Rokhsar DS, Satoh N, Holland PW: The amphioxus genome illuminates vertebrate origins and cephalochordate biology. Genome Res 2008, 18:1100-1111.

52. Minguillon C, Garcia-Fernandez J: Genesis and evolution of the evx and Mox genes and the extended Hox and ParaHox gene clusters. Genome Biol 2003, 4:R12.

53. Ryan JF, Mazza ME, Pang K, Matus DQ, Baxevanis AD, Martindale MQ: Prebilaterian origins of the hox cluster and the hox code: evidence from the sea anemone. Nematostella vectensis. PLOS ONE 2007, 2:e153.

54. Meyer NP, Boyle MJ, Martindale MQ, Seaver EC: A comprehensive fate map by intracellular injection of identified blastomeres in the marine polychaete Capitella teleta. EvoDevo 2010, 1:8.

55. Slightom $J$, Blechl AE, Smithies $O$ : Human fetal G gamma- and A gamma-globin genes: complete nucleotide sequences suggest that DNA can be exchanged between these duplicated genes. Cell 1980, 21:627-638.

56. Smithies $\mathrm{O}$, Powers PA: Gene conversions and their relation to homologous chromosome pairing. Philos Trans R Soc Lond B Biol Sci 1986, 312:291-302

57. McDougall C, Korchagina N, Tobin JL, Ferrier DE: Annelid Distal-less/Dlx duplications reveal varied post-duplication fates. BMC Evol Biol 2011, $11: 241$.

58. Woodruff JB, Mitchell BJ, Shankland M: Hau-Pax3/7A is an early marker of mesoderm involved in segmental morphogenesis, nephridial development, and body cavity formation. Dev Biol 2007, 306:824-837.

59. Wang W, Zhong J, Wang YQ: Comparative genomic analysis reveals the evolutionary conservation of Pax gene family. Genes Genet Syst 2010, 85:193-206.

60. Frigerio G, Burri M, Bopp D, Baumgartner S, Noll M: Structure of the segmentation gene paired and the Drosophila PRD gene set as part of a gene network. Cell 1986, 47:735-746.

61. Chipman AD, Akam M: The segmentation cascade in the centipede Strigamia maritima: involvement of the Notch pathway and pair-rule gene homologues. Dev Biol 2008, 319:160-169.

62. Dequeant ML, Pourquie O: Segmental patterning of the vertebrate embryonic axis. Nat Rev Genet 2008, 9:370-382.

63. Stollewerk A, Schoppmeier M, Damen WG: Involvement of Notch and Delta genes in spider segmentation. Nature 2003, 423:863-865.

64. Carroll SB, Laughon A, Thalley BS: Expression, function, and regulation of the hairy segmentation protein in the Drosophila embryo. Genes Dev $1988,2: 883-890$.

65. Sommer RJ, Tautz D: Involvement of an orthologue of the Drosophila pair rule gene hairy in segment formation of the short germ band embryo of Tribolium (Coleoptera). Nature 1993, 361:448-450.

66. Dormand $\mathrm{E}-\mathrm{L}$, Brand $\mathrm{AH}$ : Runt determines cell fates in the Drosophila embryonic CNS. Development 1998, 125:1659-1667.

67. Kalev-Zylinska M, Horsfield J, Flores M, Postlethwait J, Vitas M, Baas A, Crosier $P$, Crosier K: Runx1 is required for zebrafish blood and vessel development and expression of a human RUNX1-CBF2T1 transgene advances a model for studies of leukemogenesis. Development 2002, 129:2015-2030.

68. Chen A, de Nooij J, Jessell T: Graded activity of transcription factor Runx3 specifies the laminar termination pattern of sensory axons in the developing spinal cord. Neuron 2006, 49:395-408.

69. Zagami CJ, Zusso M, Stifani S: Runx transcription factors: lineage-specific regulators of neuronal precursor cell proliferation and post-mitotic neuron subtype development. J Cell Biochem 2009, 107:1063-1072.

70. Denes AS, Jekely G, Steinmetz PR, Raible F, Snyman H, Prud'homme B, Ferrier DE, Balavoine G, Arendt D: Molecular architecture of annelid nerve cord supports common origin of nervous system centralization in Bilateria. Cell 2007, 129:277-288.

71. Goulding MD, Chalepakis G, Deutsch U, Erselius JR, Gruss P: Pax-3, a novel murine DNA binding protein expressed during early neurogenesis. $E M B O$ J 1991, 10:1135-1147.
72. Jostes B, Walther C, Gruss P: The murine paired box gene, Pax-7, is expressed specifically during the development of the nervous and muscular system. Mech Dev 1991, 33:27-38.

73. de Rosa R, Prud'homme B, Balavoine G: Caudal and even-skipped in the annelid Platynereis dumerilii and ancestry of posterior growth. Evol Dev 2005, 7:574-587.

74. Song MH, Huang FZ, Chang GY, Weisblat DA: Expression and function of an even-skipped homolog in the leech Helobdella robusta. Development 2002, 129:3681-3692.

75. Sordino P, Duboule D, Kondo T: Zebrafish Hoxa and Evx-2 genes: cloning, developmental expression and implications for the functional evolution of posterior Hoxgenes. Mech Dev 1996, 59:165-175.

76. Ruiz i Altaba A, Melton DA: nvolvement of the Xenopus homeobox gene Xhox3 in pattern formation along the anterior-posterior axis. Cell 1989, 57:317-326.

77. Ahringer J: Posterior patterning by the Caenorhabditis elegans evenskipped homolog vab-7. Genes Dev 1996, 10.

78. Brown SJ, Parrish JK, Beeman RW, Denell RE: Molecular characterization and embryonic expression of the even-skipped orthologue of Tribolium casaneum. Mech Dev 1997, 61:165-173.

79. Copf T, Rabet N, Celniker SE, Averof M: Posterior patterning genes and the identification of a unique body region in the brine shrimp Artemia franciscana. Development 2003, 130:5915-5927.

80. Patel NH, Condron BG, Zinn K: Pair-rule expression patterns of evenskipped are found in both short-and long-germ beetles. Nature 1994, 367:429-434.

81. Doe C, Smouse D, Goodman CS: Control of neuronal fate by the Drosophila segmentation gene even-skipped. Nature 1988, 333:376-378.

82. Esmaeili B, Ross JM, Neades C, Miller DM, Ahringer J: The C. elegans evenskipped homologue, vab-7, specifies DB motoneurone identity and axon trajectory. Development 2002, 129:853-862

83. Ruiz i Altaba A: Neural expression of the Xenopus homeobox gene Xhox3: evidence for a patterning neural signal that spreads through the ectoderm. Development 1990, 108:595-604.

84. Bastian H, Gruss P: A murine even-skipped homologue, Evx 1, is expressed during early embryogenesis and neurogenesis in a biphasic manner. EMBO J 1990, 9:1839-1852.

85. Seebald JL, Szeto DP: Zebrafish eve1 regulates the lateral and ventral fates of mesodermal progenitor cells at the onset of gastrulation. Dev Biol 2011, 349:78-89.

86. Saudemont A, Dray N, Hudry B, Le Gouar M, Vervoort M, Balavoine G: Complementary striped expression patterns of NK homeobox genes during segment formation in the annelid Platynereis. Dev Biol 2008, 317:430-443.

87. Janssen R, Le Gouar M, Pechmann M, Poulin F, Bolognesi R, Schwager EE, Hopfen C, Colbourne JK, Budd GE, Brown SJ, Prpic NM, Kosiol C, Vervoort M, Damen WG, Balavoine G, McGregor AP: Conservation, loss, and redeployment of Wnt ligands in protostomes: implications for understanding the evolution of segment formation. BMC Evolutionary Biology 2010, 10:374.

88. Dray N, Tessmar-Raible K, Le Gouar M, Vibert L, Christodoulou F, Shipany K, Guillou A, Zantke J, Snyman H, Behague J, Vervoort M, Arendt D, Balavoine G: Hedgehog signaling regulates segment formation in the annelid Platynereis. Science 2010, 329:339-342.

doi:10.1186/2041-9139-3-8

Cite this article as: Seaver et al:: Expression of the pair-rule gene homologs runt, Pax3/7, even-skipped- 1 and even-skipped- 2 during larval and juvenile development of the polychaete annelid Capitella teleta does not support a role in segmentation. EvoDevo 2012 3:8. 